\title{
Article
}

\section{The emergence of extramural cemeteries in Neolithic southeast Europe: a formally modeled chronology for Cernica, Romania}

Strattion, Susan, Griffiths, Seren, Kogălniceanu, Raluca and

Simalcsik, Angela

Available at http://clok.uclan.ac.uk/23070/

Strattion, Susan, Griffiths, Seren ORCID: 0000-0001-5168-9897, Kogălniceanu, Raluca and Simalcsik, Angela (2019) The emergence of extramural cemeteries in Neolithic southeast Europe: a formally modeled chronology for Cernica, Romania. Radiocarbon, 61 (1). pp. 319-346. ISSN 0033-8222

It is advisable to refer to the publisher's version if you intend to cite from the work. http://dx.doi.org/10.1017/RDC.2018.34

For more information about UCLan's research in this area go to http://www.uclan.ac.uk/researchgroups/ and search for <name of research Group>.

For information about Research generally at UCLan please go to http://www.uclan.ac.uk/research/

All outputs in CLoK are protected by Intellectual Property Rights law, including Copyright law. Copyright, IPR and Moral Rights for the works on this site are retained by the individual authors and/or other copyright owners. Terms and conditions for use of this material are defined in the policies page. 


\section{THE EMERGENCE OF EXTRAMURAL CEMETERIES IN NEOLITHIC SOUTHEAST EUROPE: A FORMALLY MODELED CHRONOLOGY FOR CERNICA, ROMANIA}

\author{
Susan Stratton ${ }^{1} \cdot$ Seren Griffiths $^{2} \cdot$ Raluca Kogălniceanu $^{3}$ - Angela Simalcsik ${ }^{4}$ • \\ Alexandru Morintz ${ }^{3} \cdot$ Cristian Eduard Ştefan ${ }^{3} \cdot$ Valentin Dumitraşcu $^{3} \bullet$ \\ Christopher Bronk Ramsey ${ }^{5}$ Olaf Nehlich ${ }^{6}$ - Nancy Beavan ${ }^{7}$ Dušan Borić ${ }^{8 *}$ • \\ Alasdair Whittle ${ }^{1}$
}

${ }^{1}$ Department of Archaeology and Conservation, SHARE, Cardiff University, John Percival Building, Colum Drive, Cardiff CF10 3EU, United Kingdom.

${ }^{2}$ The School of Forensic and Applied Sciences, University of Central Lancashire, Preston, Lancashire, PR1 2HE, United Kingdom.

3“Vasile Pârvan” Institute of Archaeology, Romanian Academy, 11 Henri Coandă Street, Bucharest, Romania.

4“Olga Necrasov" Centre of Anthropology, Romanian Academy - Iaşi branch, 2 Th. Codrescu Street, Iaşi, Romania.

${ }^{5}$ Oxford Radiocarbon Accelerator Unit, Research Laboratory for Archaeology and the History of Art, Dyson Perrins Building, University of Oxford, Oxford OX1 3QY, United Kingdom.

${ }^{6}$ Department of Anthropology, University of British Columbia, 6303 N.W. Marine Drive, Vancouver, BC, V6T 1Z1 Canada.

${ }^{7}$ Department of Anatomy and Structural Biology, University of Otago, School of Medical Sciences, PO Box 913, Dunedin 9054, New Zealand.

${ }^{8}$ The Italian Academy for Advanced Studies in America, Columbia University, 1161 Amsterdam Avenue, New York, NY 10027, USA.

\begin{abstract}
The emergence of separate cemeteries for disposal of the dead represents a profound shift in mortuary practice in the Late Neolithic of southeast Europe, with a new emphasis on the repeated use of a specific space distinct from, though still often close to, settlements. To help to time this shift more precisely, this paper presents 25 dates from 21 burials in the large cemetery at Cernica, in the Lower Danube valley in southern Romania, which are used to formally model the start, duration of use and end of the cemetery. A further six dates were obtained from four contexts for the nearby settlement. Careful consideration is given to the possibility of environmental and dietary offsets. The preferred model, without freshwater reservoir offsets, suggests that use of the Cernica cemetery probably began in 5355-5220 cal BC (95\% probability) and ended in 5190-5080 cal BC (28\% probability) or 5070-4940 $(67 \%)$. The implications of this result are discussed, including with reference to other cemeteries of similar age in the region, the nature of social relations being projected through mortuary ritual, and the incorporation of older, Mesolithic, ways of doing things into Late Neolithic mortuary practice.
\end{abstract}

KEYWORDS: Bayesian modeling, burial, Cernica, Lower Danube, Neolithic.

\section{INTRODUCTION}

\section{Emergence of Extramural Cemeteries in Neolithic Southeast Europe}

Evidence of inhumation before the first half of the fifth millennium cal BC in southeast Europe - often otherwise known as the Late Neolithic - is regionally patchy. In Mesolithic burial practices, represented primarily by finds from sites in the Danube Gorges, people were laid mostly in supine extended positions (Borić 2011, 2016). With the appearance of Neolithic communities in southeast Europe from the middle of the seventh millennium cal BC onward, formal mortuary custom changed, placing the body in a flexed position on a lateral left or right side, a tradition considered to originate in the Near East (Bonsall 2007:54; Borić 2015). Burials from the seventh to late sixth millennia cal $\mathrm{BC}$ across southeast Europe - conventionally the Early and Middle Neolithic - have been found exclusively in settlements, normally in individual graves, and were generally unaccompanied by grave goods (Lichter 2001; Schuster et al. 2008). The very small number of burials known from the Early and Middle Neolithic indicates that this was definitely not the only, and was unlikely to have been the primary, form of mortuary practice (Bojadzhiev 2001; Lichter 2001; Kogălniceanu 2009; Borić 2015).

\footnotetext{
*Corresponding author. Email: db2128@columbia.edu.
} 


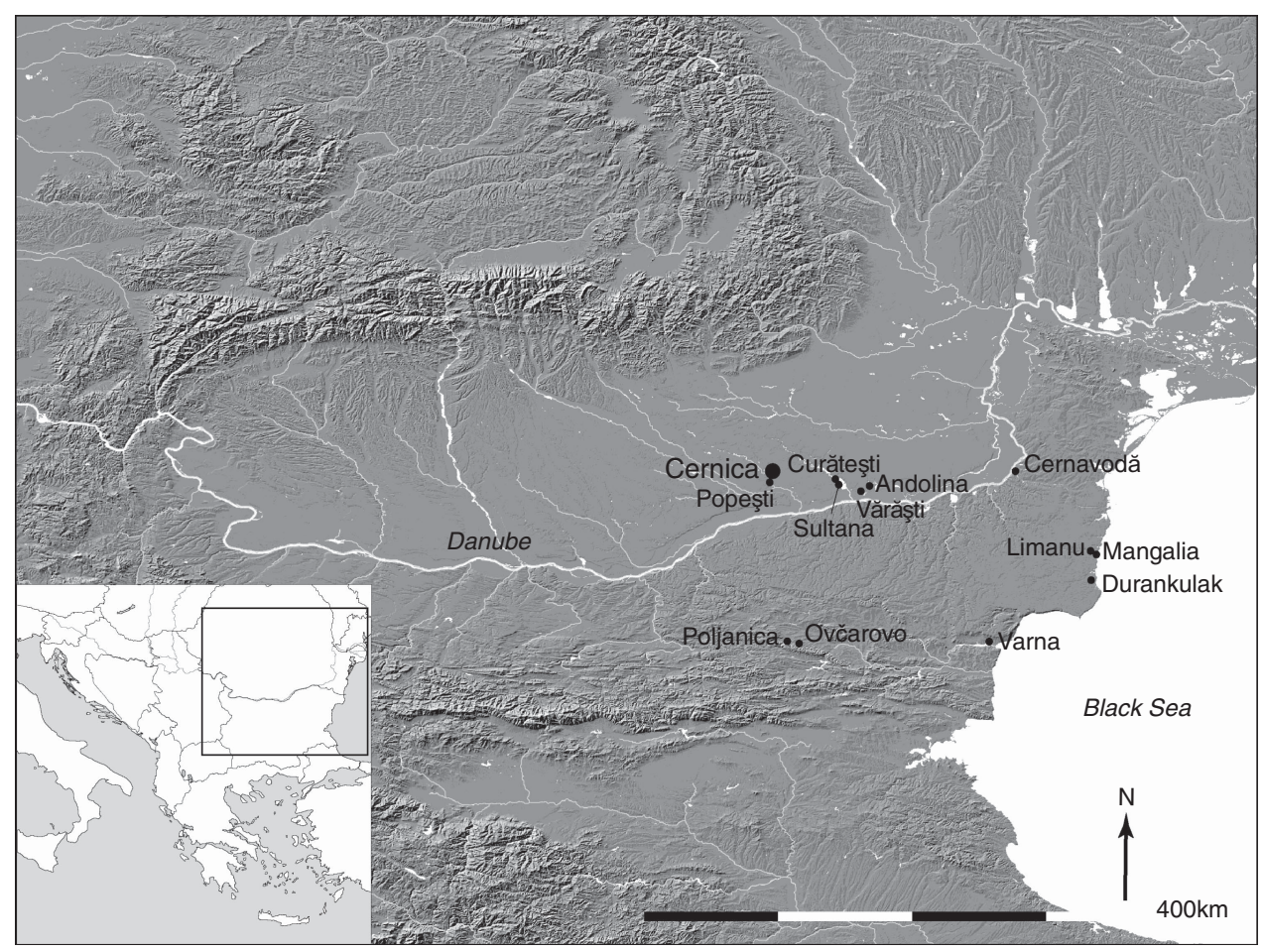

Figure 1 Location of Cernica and other late-6th and 5th-millennium cal BC cemeteries mentioned in the text.

The Late Neolithic and Early Copper Age in the eastern Balkans saw the quite widespread emergence of large, extramural cemeteries in several contexts (Figure 1). In Muntenia (Kogălniceanu 2012; Lazăr 2012), the sites of Cernica (Comşa and Cantacuzino 2001; Kogălniceanu 2005), Sultana-Valea Orbului (Şerbănescu 2002; Şerbănescu et al. 2007, 2008), Popeşti (Şerbănescu 1999), Andolina (Comşa 1974a, 1974b), Vărăşti-Grădiştea Ulmilor / "Boian A" (Comşa 1974a; Kogălniceanu 2012 with bibliography) and Curăteşti (Şerbănescu and Soficaru 2006; Şerbănescu and Cristache 2011) had varying numbers of burials: 378, 253, 16, 9, 14-18, and 20, respectively. In Dobrudza, the region situated between the Lower Danube and the Black Sea, Durankulak had around 600 Hamangia burials (Todorova 2002), and Cernavodă 556 (Morintz et al. 1955; Berciu and Morintz 1957, 1959; Berciu et al. 1959, 1961; Necrasov et al. 1990). There are two more Hamangia cemeteries discovered in this area, at Mangalia and Limanu, unfortunately with little precise information (Kogălniceanu 2012 and references therein). In the Poljanica culture of northeast Bulgaria, somewhat smaller cemeteries were found adjacent to the sites of Poljanica, Radingrad, and Ovčarovo, with 25 (Todorova 1982), 21 (Todorova 1982), and 3 burials (Todorova et al. 1983). The earlier practice of predominantly flexed burials continued in most of these cemeteries where only left-flexed burials were found. The burials at Cernica (and at other sites such as Cernavodă and Durankulak) differ, as the placement of the body in an extended supine position dominates, with the body lying on the back, with the face up, and with less than one-tenth of burials placed in right- or left-flexed positions. These cemeteries are also distinguished by being amongst the earliest — in terms of material culture — and the largest in terms of numbers of graves.

The emergence of separate cemeteries for disposal of the dead represents a profound shift, with an emphasis on the repeated use of a specific space distinct from, though still often close to, 
settlements; there are potentially significant new relations between the living and the dead. It is the timing of this shift in southeast Europe that we aim to help to establish in this paper. We present the results of a recent project to radiocarbon date the cemetery of Cernica, as well as its adjacent settlement, found $50 \mathrm{~m}$ to its southwest. The project has used 25 dates from 21 burials to formally model the start, duration of use and end of the cemetery, and a further six dates were obtained for the settlement. Multi-isotope dietary signatures were also obtained.

\section{Cernica Cemetery and Settlement}

The cemetery at Cernica, $10 \mathrm{~km}$ southeast of Bucharest, was found during the excavation of a medieval monastery located on the shores of Lake Cernica (Comşa and Cantacuzino 2001; Kogălniceanu 2009). The lake was formed by the damming of the River Colentina in the 1960s and the cemetery is located on a former terrace of the Colentina, which now forms a projection into the lake. Settlement remains were also discovered in the area (Figure 2). The settlement is located southwest of the cemetery, farther inland. It was not investigated completely and its actual limits are not known as a forest prevented the excavation much farther to the southwest. Test pits were placed in the area of the forest and the results indicated that the settlement extended in a southwesterly direction. The research carried out in the settlement between 1961 and 1967 led to the identification of several layers, culturally attributed to the Dudeşti culture (last phase-Cernica) and Boian culture (first phase-Bolintineanu). Sporadic discoveries indicated also the presence of the second phase of the Boian culture (Goloviţa). A report up to 1968 indicated the discovery of 102 pits, of which 34 were attributed to the Dudeşti culture, 65 to the Boian culture-Bolintineanu phase and only three to the Boian culture-Goloviţa phase. Some of the pits were reported as having been used as habitation structures, but no further details are available at present (Cantacuzino and Morintz 1968:9-10).

The cemetery and settlement were excavated by Gheorghe Cantacuzino and Sebastian Morintz from 1961 to 1974 , uncovering a minimum of $378^{1}$ burials from an area of roughly $4550 \mathrm{~m}^{2}$. The cemetery is formed of two main groups or clusters, north (with 174 burials) and south (204 burials), with some outliers to the east and west and in the centre, which could possibly be considered a separate group (Figure 3) (Ursulescu and Kogălniceanu 2007). However, it seems more likely that these central burials are outliers from the northern and southern nuclei. There is nothing to indicate any specific practice relating exclusively to either nucleus, nor to tell us whether they were in use at the same time. Inconsistencies in the site records have been noted (Kogălniceanu 2009). The whereabouts of some of the grave goods from the cemetery have been elusive until recently, making sampling for dating of bone or antler artefacts from the graves impossible. However, recent archive work has been successful in relocating part of the assemblage, which has now been published (Ciocănel [Vintilă] 2015; Mărgărit and Vintilă 2015).

The lack of ceramic finds (only five graves contained any pottery) makes the usual culturehistorical assignations difficult. The cemetery appears to belong to the Late Neolithic Boian culture (Cantacuzino and Morintz 1963; Comşa 1975), though this has been disputed and an earlier date, prior to the start of the Boian culture, was proposed (Comşa 1992; Comşa and Cantacuzino 2001; Şerbănescu 2015).

\footnotetext{
${ }^{1}$ The exact number of burials is not certain due to discrepancies between the annual site records, excavators' reports, and the final publication. The total number of burials in the site monograph is claimed to be 374 (Comşa and Cantacuzino 2001), yet the number of burials described is 378 . Two further burials have been found when recently compiling the site plan, raising the total number to 380 (Kogălniceanu 2009:13). One of the burials recorded, 108bis, does not appear on the plan and there is no drawing of it. This total includes Burial 356, which is the burial found on the settlement. Therefore, it is most likely that 378 individuals were buried in the cemetery (Kogălniceanu 2009).
} 


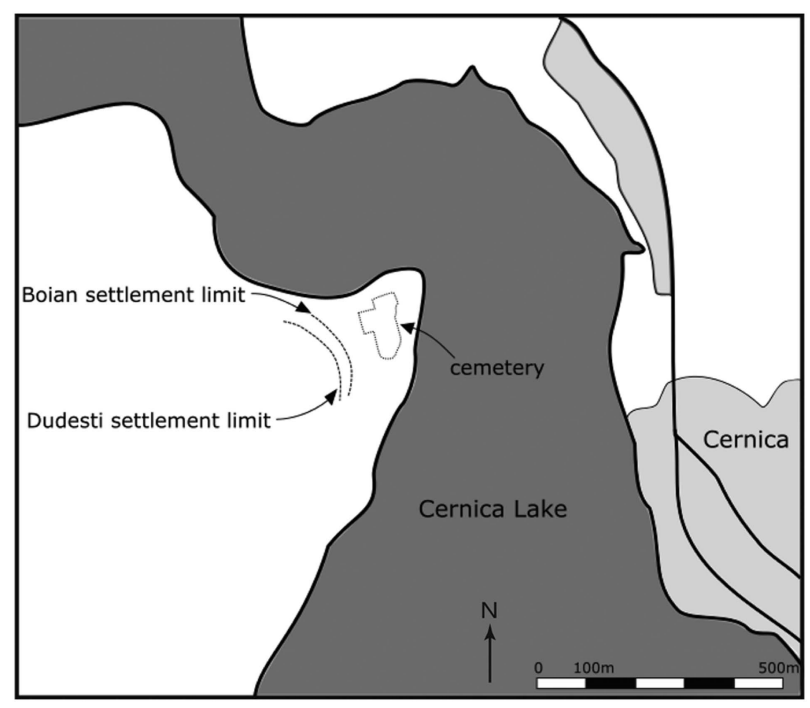

Figure 2 Location of excavation area on Cernica Lake (adapted after Cantacuzino and Morintz 1968: Figure 1).

Very few stratigraphic relationships are present on the site. The suggestion (Comşa and Cantacuzino 2001) that the extended burials as a group could pre-date the flexed/crouched ones (Figure 4) was based on a few examples (Burials 47 and 48 [Figure 5]; Burials 139 and 140; Burials 153 and 145; Burials 191C and 191D) where an extended burial was cut or overlain by an individual in a flexed position (Comşa and Cantacuzino 2001). However, the documentary evidence indicates that in the case of Burials 139 and 140, the supine extended skeleton may have overlain the flexed one (Kogălniceanu 2009). There is currently no clear evidence to suggest that these two types of burial could not have been in use contemporaneously. Flexed/ crouched burials number only 20 out of the total of 378 , although the majority of these (15) would probably more accurately be described as flexed as the lower limbs at the knees are bent but not drawn up toward the chest. Some of these burials could also be described as extended supine with slightly flexed lower limbs. Their distribution across the site is even, and as such does not appear to represent a specific developmental phase. The orientation of both extended supine and left- and right-flexed burials from Cernica is mainly west-east.

In some of the burials at Cernica, perforated red deer canines were found as ornaments, a practice considered to be part of older Palaeolithic and Mesolithic traditions (Borić 2015). Burials 28, 34, 171, and 173, dated in this project (see below), all had red deer canines in the skull area, possibly from strings of beads. These were usually found along with other beads of Dentalium, Spondylus (Figure 6), malachite, or copper. Shell beads in bi-lobed or tri-lobed form (found in our dated Burials 34, 188, 194, and 292: Figure 5), along with the bone idol-pendant from our dated Burial 37 (Figure 6:3), have been considered typical of the Boian culture. A Spondylus armband was found in Burial 141 (Figure 6:6) (failed sample in this project) and Glycymeris armbands were in the dated Burials 188 and 267 (Figure 6:7, 6:9-10; see also Figures 4 and 5). While the small flint pieces (microliths) identified in some of the graves from the cemetery (in the undated Burials 84, 97, 156, 227, and 241A) are thought to be Dudeşti material culture (which precedes Boian), other artefacts are typical Boian (those already mentioned above, or the Spondylus valve placed on the pubis from the undated Burials 43, 266, and 314). Unfortunately, at this point, there is still very little known about the material culture of the 


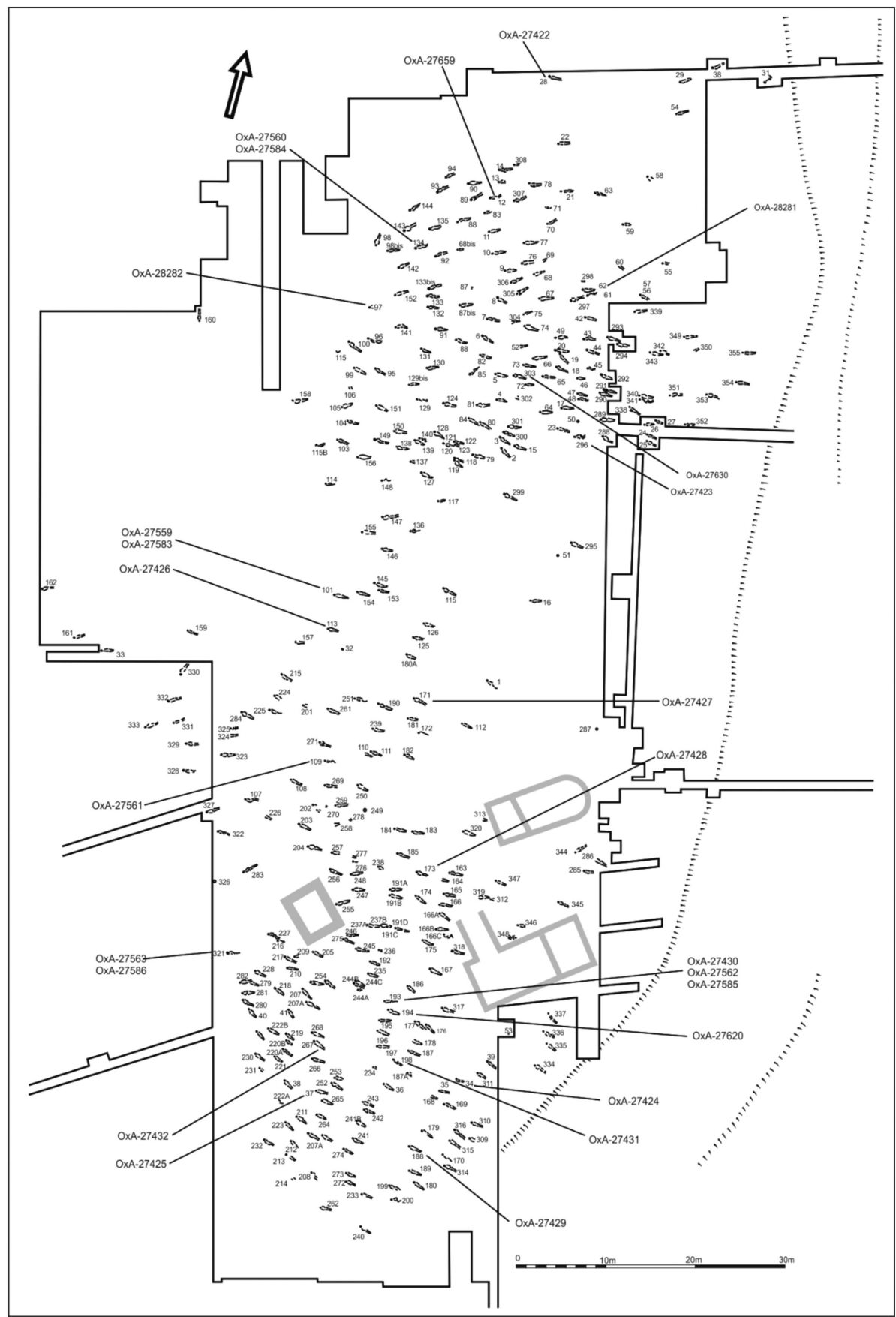

Figure 3 Plan of Cernica cemetery, with sampled burials marked (redrawn after Comşa and Cantacuzino 2001:Plate 37).

Dudeşti taxonomic unit. Copper or malachite green beads found in our dated Burial 267 (Figure 6:11) and Burial 29 (failed sample) may represent some of the earliest uses of copper in Europe (cf. Borić 2009; Rosenstock et al. 2016). 

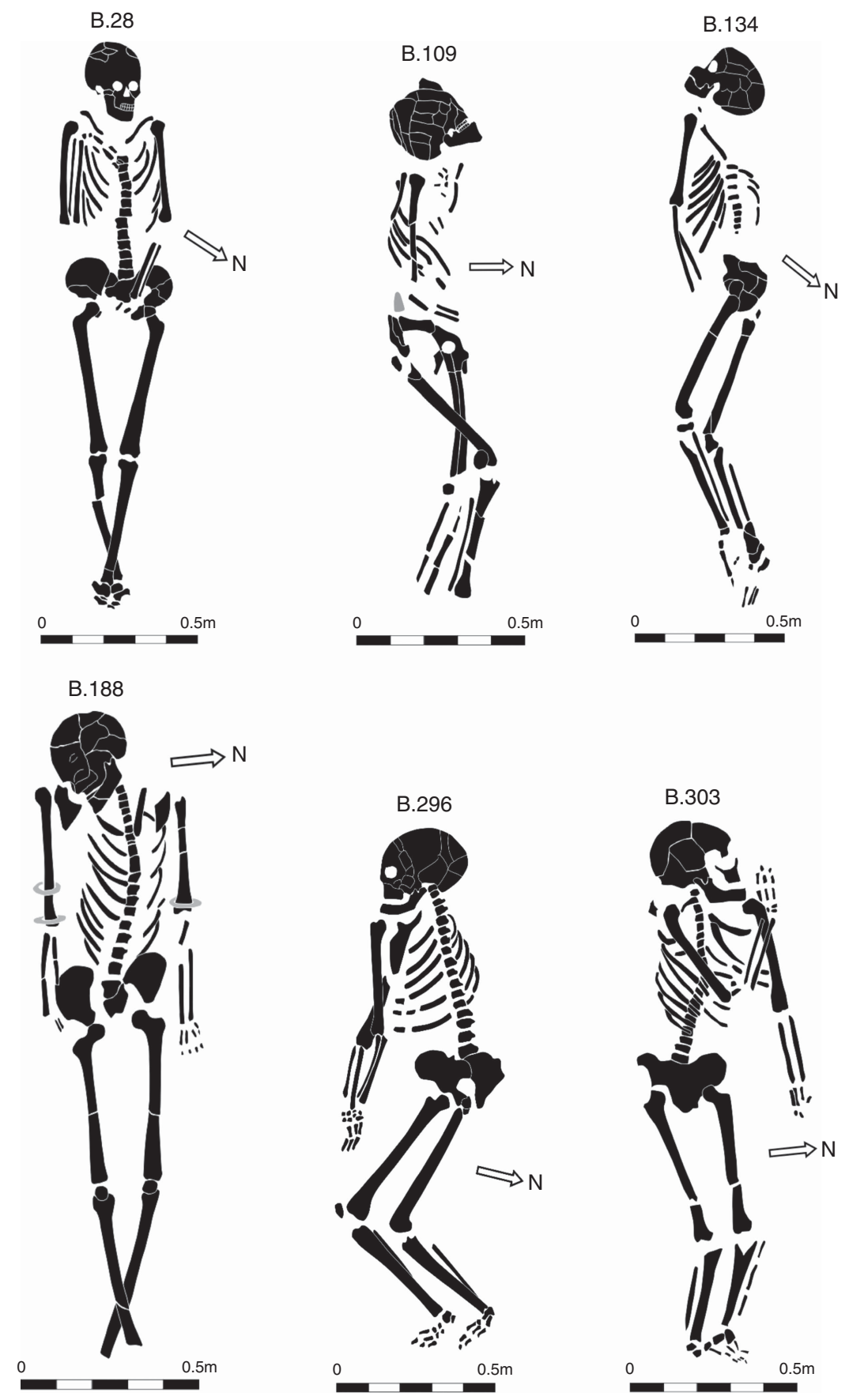

Figure 4 Burials 28, 109, 134, 188, 296, and 303 at Cernica (redrawn after Comşa and Cantacuzino 2001:Plates III, XI, XIV, XIX, XXX, and XXXI).

Finds from the settlement indicate the presence of both Boian and late Dudeşti material. A single burial was found in the settlement, possibly dating to the Dudeşti culture (Schuster et al. 2008). However, the supine extended burial in question, Burial 356, has no stratigraphic 


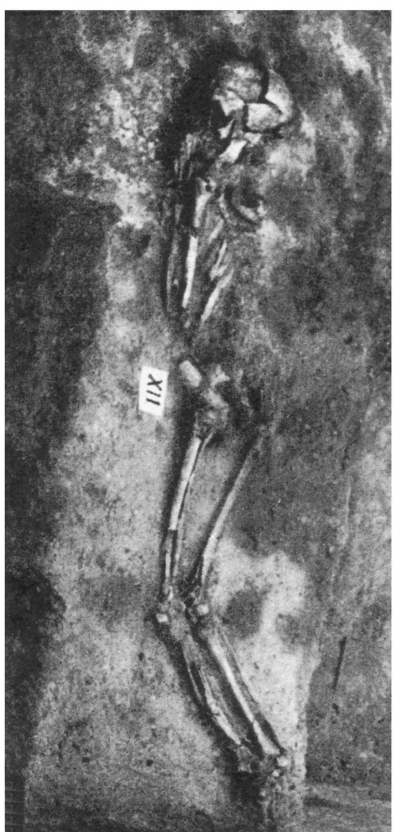

B. 12

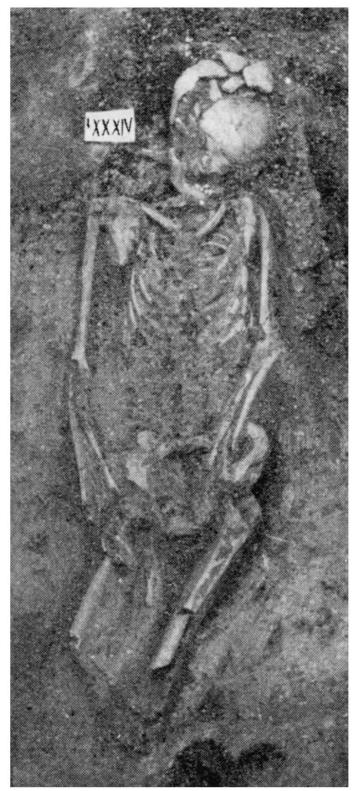

B. 34

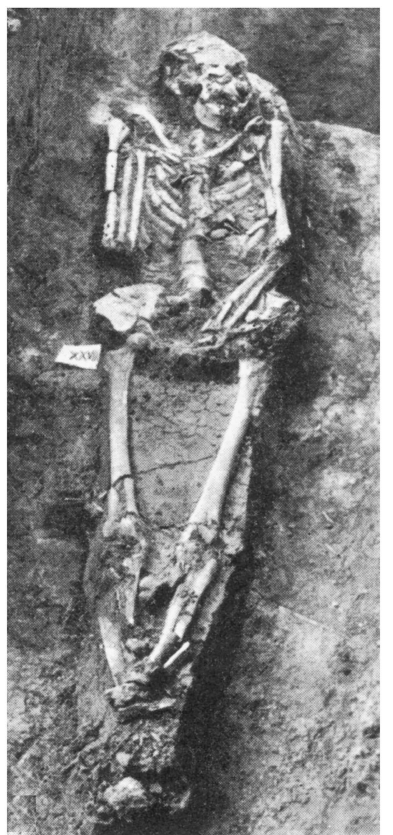

B. 28

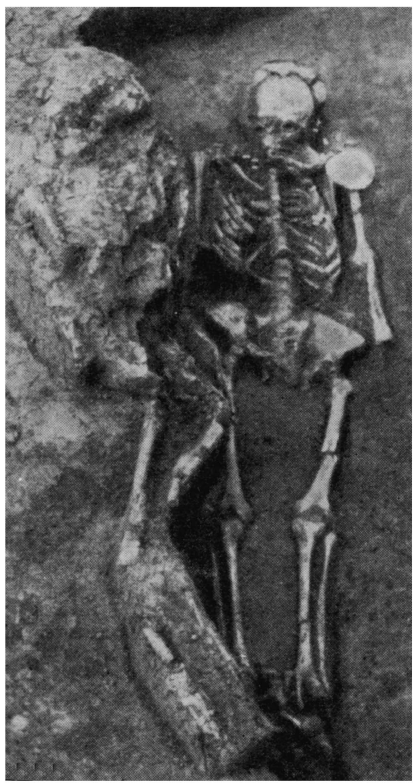

B. 47 and 48

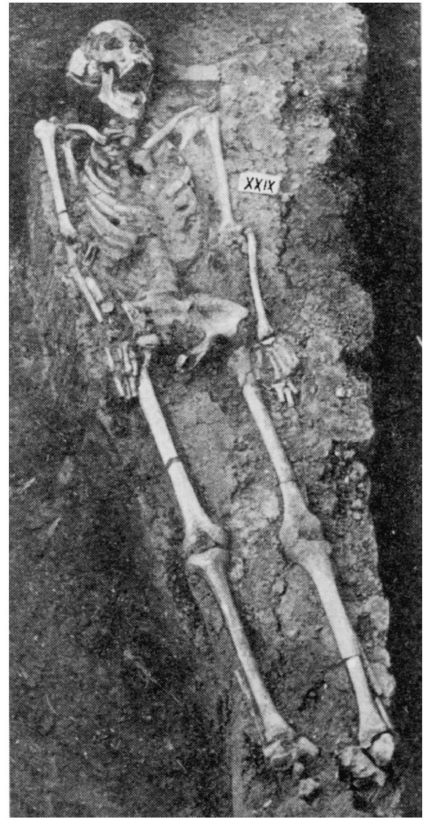

B. 29

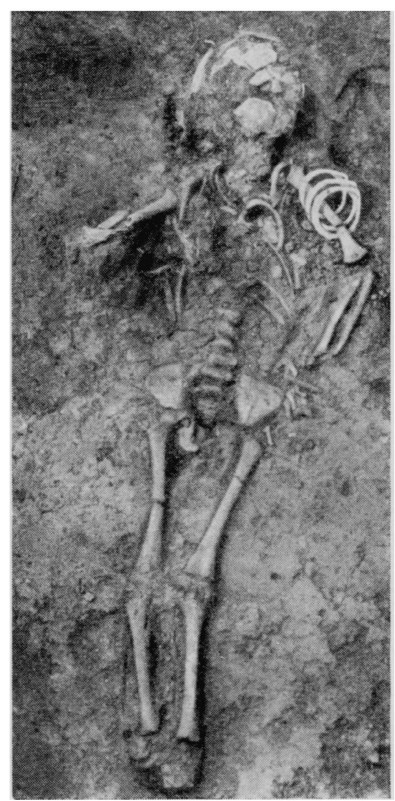

B. 88bis

Figure 5 Burials 12, 28, 29, 34, 47, 48, and 88bis at Cernica (after Ciocănel [Vintilă] 2015).

information and had no grave goods (Comşa and Cantacuzino 2001). The bone was poorly preserved, no anthropological assessment has been made (Kogălniceanu 2009), nor was the material available for dating. 


\section{S Stratton et al.}
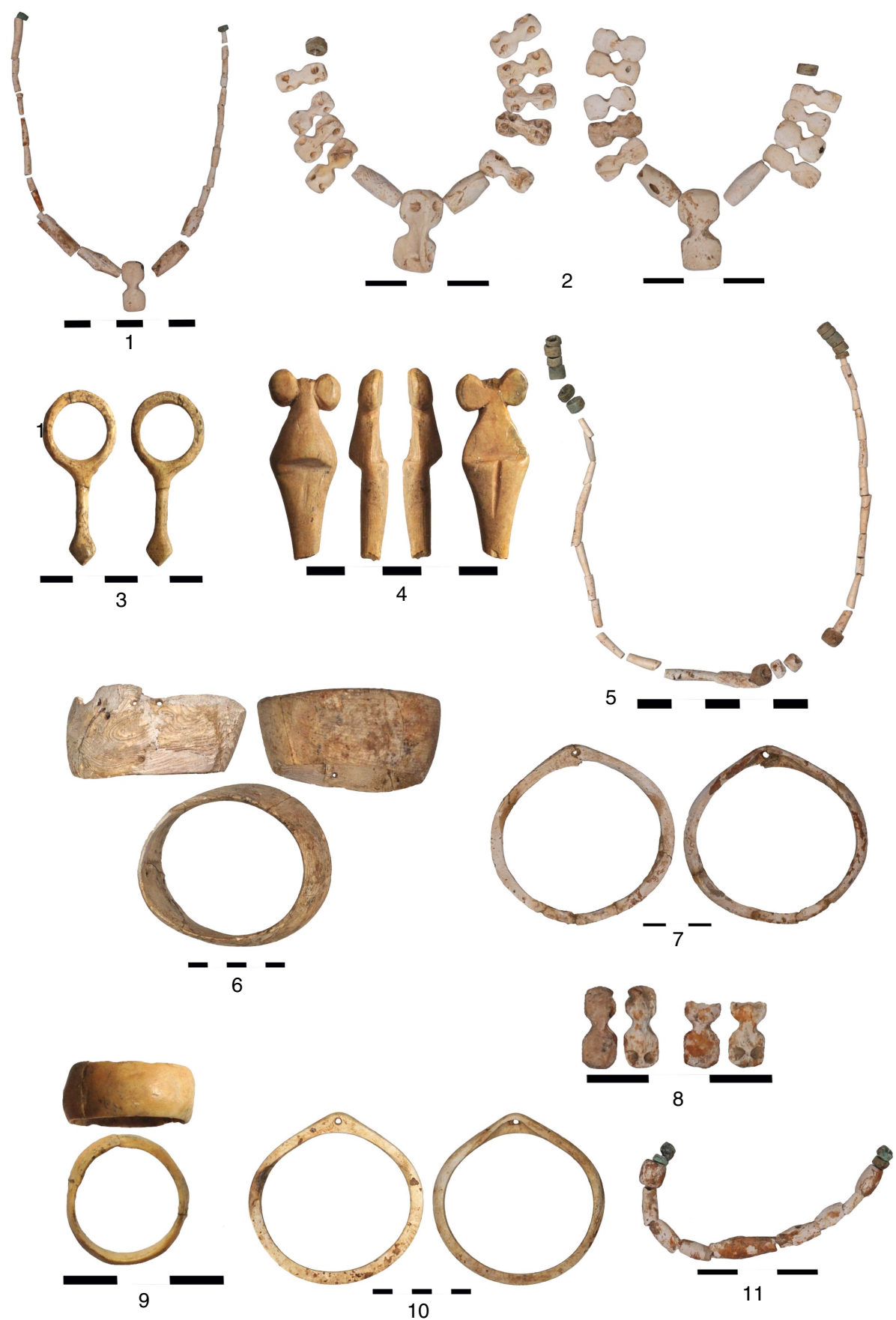

Figure 6 Objects of personal adornment and grave offerings found in some of the dated burials from Cernica. 1: Burial 29, Spondylus beads; 2: Burial 34, Spondylus beads/pendants; 3 : Burial 37, bone pendant; 4-5: Burial 101, bone figurine and a necklace of Dentalium and diskshaped stone beads; 6: Burial 141, Spondylus armband; 7-8: Burial 188, Glycymeris armband and pendants; 9-11: Burial 267, Glycymeris armband, pendant and barrel-shaped beads. 
Table 1 Dated samples from Cernica. The $\delta^{13} \mathrm{C}$ and $\delta^{15} \mathrm{~N}$ values were measured at ORAU, the $\delta^{34} \mathrm{~S}$ were measured using the protocol of Nehlich et al. (2010); see online supplement for more information. The Poznań measurement had an average freshwater dietary offsets (24 \pm 14$)$ applied in subsequent modeling.

\begin{tabular}{|c|c|c|c|c|c|c|c|c|c|c|c|c|c|c|c|}
\hline Lab code & Sample number and details & $\begin{array}{l}\text { Used } \\
(\mathrm{mg})\end{array}$ & $\begin{array}{l}\text { Yield } \\
(\mathrm{mg})\end{array}$ & $\begin{array}{l}\% \\
\text { yield }\end{array}$ & $\% \mathrm{C}$ & $\delta^{13} \mathrm{C}$ & $\delta^{15} \mathrm{~N}$ & $\delta^{34} \mathrm{~S}$ & $\begin{array}{l}\mathrm{C}: \mathrm{N} \\
\text { atomic } \\
\text { ratio }\end{array}$ & $\begin{array}{l}\text { Result } \\
\text { (BP) }\end{array}$ & $\begin{array}{l}\text { Calibrated } \\
{ }^{14} \mathrm{C} \text { date }(95 \% \\
\text { confidence; } \mathrm{BC})\end{array}$ & $\begin{array}{l}\text { FRUITS \% } \\
\text { estimated } \\
\text { freshwater fish } \\
\text { dietary } \\
\text { contribution } \\
\text { (model 1: C, N) }\end{array}$ & $\begin{array}{l}\text { FRUITS \% } \\
\text { estimated } \\
\text { freshwater fish } \\
\text { dietary } \\
\text { contribution } \\
\text { (model 2: C, N, S) }\end{array}$ & $\begin{array}{l}\text { Terrestrial- } \\
\text { no correction-- } \\
\text { preferred posterior } \\
\text { density estimate } \\
(95 \% \text { probability; } \\
\text { cal BC) }\end{array}$ & $\begin{array}{l}\text { Offset-correction } \\
\text { using FRUITS } \\
\text { model 1-posterior } \\
\text { density estimate } \\
(95 \% \\
\text { probability; cal BC) }\end{array}$ \\
\hline \multicolumn{16}{|c|}{ Results from the cemetery } \\
\hline OxA-27422 & $\begin{array}{l}\text { Sample } 3 \text {, bone, Homo sapiens left humerus } \\
\text { from Burial } 28 \text {, from trench } 9 \text {, northern } \\
\text { area of cemetery. Male, estimated }<30 \text { for } \\
\text { this project. }{ }^{2} \text { Supine extended burial } \\
\text { containing deer teeth, an axe and malachite } \\
\text { beads. }\end{array}$ & 630 & 6.53 & 1 & 42.1 & $1-19.9$ & 9.5 & - & 3.2 & $6149 \pm 35$ & $5215-5000$ & $14.0 \pm 8.7$ & - & $5220-5020$ & $5080-4930$ \\
\hline OxA-27423 & $\begin{array}{l}\text { Sample 26, bone, Homo sapiens left femur } \\
\text { from Burial 296, trench 26, northern area } \\
\text { of cemetery. Female, } c .40 \text {. Right-flexed } \\
\text { burial, no grave goods noted. Discrete } \\
\text { glued repair at break away from sample } \\
\text { location. }\end{array}$ & 620 & 4.94 & 0.8 & 42 & -19.7 & 9.6 & - & 3.2 & $6266 \pm 34$ & $5325-5075$ & $14.2 \pm 8.7$ & - & $5310-5080$ & $5140-4970$ \\
\hline OxA-27424 & $\begin{array}{l}\text { Sample 5, bone, Homo sapiens left ulna } \\
\text { from Burial 34, from trench 13, southern } \\
\text { area of cemetery. Female, 16-18. Supine } \\
\text { extended burial containing Spondylus (bi- } \\
\text { lobed, barrel), animal teeth and malachite } \\
\text { beads. }\end{array}$ & 650 & 5.11 & 0.8 & 41.8 & $8-19.9$ & 10.4 & - & 3.2 & $6232 \pm 33$ & $5305-5065$ & $15.9 \pm 9.4$ & - & $5295-5070$ & $5130-4955$ \\
\hline OxA-27425 & $\begin{array}{l}\text { Sample 6, bone, Homo sapiens superior } \\
\text { maxillary left side fragment, from Burial } \\
37 \text {, trench } 12 \text {, southern area of cemetery. } \\
\text { Identified as female, } 15-16 \text {, for this project. } \\
\text { Supine extended burial containing a bone } \\
\text { idol-pendant. }\end{array}$ & 450 & 11.4 & 2.5 & 41.4 & $4-20.4$ & 9.9 & - & 3.2 & $6092 \pm 35$ & $5210-4855$ & $14.6 \pm 8.7$ & - & $5215-4980$ & $5060-4920$ \\
\hline OxA-27426 & $\begin{array}{l}\text { Sample 13, bone, Homo sapiens left tibia } \\
\text { from Burial } 113 \text {, trench } 40 \text {, southern area } \\
\text { of cemetery. Male } c .35 \text {. Supine extended } \\
\text { burial containing greenish beads and } \\
\text { shell barrel beads. }\end{array}$ & 530 & 24.43 & 4.6 & 47.5 & $5-20.2$ & 10.7 & - & 3.2 & $6157 \pm 33$ & $5215-5010$ & $16.8 \pm 9.6$ & - & $5220-5030$ & $5085-4930$ \\
\hline
\end{tabular}

${ }^{2}$ Some of the anthropological determinations may vary from Kogălniceanu 2005 and 2009 (that used the data from the unpublished anthropological manuscript), since the sampled skeletons were reexamined by one of the authors of this paper (A.S.). 


\begin{tabular}{|c|c|c|c|c|c|c|c|c|c|c|c|c|c|c|c|}
\hline Lab code & Sample number and details & $\begin{array}{l}\text { Used } \\
(\mathrm{mg})\end{array}$ & $\begin{array}{l}\text { Yield } \\
(\mathrm{mg})\end{array}$ & $\begin{array}{l}\% \\
\text { yield }\end{array}$ & $\% \mathrm{C}$ & $\delta^{13} \mathrm{C}$ & $\delta^{15} \mathrm{~N}$ & $\delta^{34} \mathrm{~S}$ & $\begin{array}{l}\mathrm{C}: \mathrm{N} \\
\text { atomic } \\
\text { ratio }\end{array}$ & $\begin{array}{l}\text { Result } \\
\text { (BP) }\end{array}$ & $\begin{array}{l}\text { Calibrated } \\
{ }^{14} \mathrm{C} \text { date }(95 \% \\
\text { confidence; } \mathrm{BC} \text { ) }\end{array}$ & $\begin{array}{l}\text { FRUITS \% } \\
\text { estimated } \\
\text { freshwater fish } \\
\text { dietary } \\
\text { contribution } \\
\text { (model 1: C, N) }\end{array}$ & $\begin{array}{l}\text { FRUITS \% } \\
\text { estimated } \\
\text { freshwater fish } \\
\text { dietary } \\
\text { contribution } \\
\text { (model 2: C, N, S) }\end{array}$ & $\begin{array}{l}\text { Terrestrial- } \\
\text { no correction-- } \\
\text { preferred posterior } \\
\text { density estimate } \\
(95 \% \text { probability; } \\
\text { cal BC) }\end{array}$ & $\begin{array}{l}\text { Offset-correction } \\
\text { using FRUITS } \\
\text { model 1-posterior } \\
\text { density estimate } \\
(95 \% \\
\text { probability; cal BC) }\end{array}$ \\
\hline OxA-27427 & $\begin{array}{l}\text { Sample 18, bone, Homo sapiens left femur } \\
\text { from Burial 171, trench } 84 \mathrm{~B} \text {, southern area } \\
\text { of cemetery. Male, identified as } c .30 \text { for } \\
\text { this project. Supine extended burial } \\
\text { containing deer teeth. Discrete glued repair } \\
\text { at break away from sample location. }\end{array}$ & 910 & 22.7 & 2.5 & 44.9 & $9-20.1$ & 10.5 & 4.0 & 3.2 & $6121 \pm 35$ & $5210-4960$ & $16.3 \pm 9.4$ & $14.7 \pm 10.1$ & $5215-5000$ & $5065-4920$ \\
\hline OxA-27428 & $\begin{array}{l}\text { Sample 19, bone, Homo sapiens left } \\
\text { humerus (half superior part of the } \\
\text { diaphysis) from Burial } 173 \text {, trench } 84 \mathrm{~B} \text {, } \\
\text { southern area of cemetery. Female, } \\
\text { identified for this project as less than } 20 \text {. } \\
\text { Supine extended burial containing } \\
\text { perforated animal teeth. }\end{array}$ & 830 & 29.5 & 3.6 & 43.8 & -20.6 & 10.3 & 2.6 & 3.1 & $6181 \pm 35$ & $5225-5010$ & $14.1 \pm 8.5$ & $9.7 \pm 7.6$ & $5230-5040$ & $5115-4945$ \\
\hline OxA-27429 & $\begin{array}{l}\text { Sample 20, bone, Homo sapiens upper left } \\
\text { maxillary from Burial 188, trench 84B, } \\
\text { southern area of cemetery. Male, } c .35 \text {. } \\
\text { Supine extended burial containing narrow } \\
\text { shell bracelets (one Glycymeris, others } \\
\text { unknown, all three published previously as } \\
\text { Pectunculus) and shell bi-lobed and tubular } \\
\text { beads. }\end{array}$ & 990 & 54.8 & 5.5 & 44.4 & -20.8 & 11.5 & 4.0 & 3.2 & $6370 \pm 40$ & $5475-5230$ & $19.2 \pm 10.2$ & $15.5 \pm 10.0$ & $5340-5210$ & $5150-4985$ \\
\hline OxA-27431 & $\begin{array}{l}\text { Sample 23, bone, Homo sapiens left femur } \\
\text { superior part from Burial 198, trench } 86 \mathrm{~B} \text {, } \\
\text { southern area of cemetery. Female, } 35 \text {. } \\
\text { Left-extended burial containing greenish } \\
\text { beads. }\end{array}$ & 460 & 25.5 & 5.5 & 44 & -20.2 & 9.7 & 5.2 & 3.2 & $6110 \pm 35$ & $5210-4940$ & $14.3 \pm 8.7$ & $12.4 \pm 9.1$ & $5215-4995$ & $5065-4920$ \\
\hline OxA-27432 & $\begin{array}{l}\text { Sample 24, bone, Homo sapiens left tibia } \\
\text { from Burial } 267 \text {, trench 101B, southern } \\
\text { area of cemetery. Female, 35-40. Supine } \\
\text { extended burial containing narrow } \\
\text { Glycymeris bracelet, bone ring, Spondylus } \\
\text { and copper beads. }\end{array}$ & 9800 & 52.8 & 0.5 & 43.8 & -20.5 & 10.2 & 3.1 & 3.2 & $6284 \pm 34$ & $5330-5210$ & $14.9 \pm 9.0$ & $10.8 \pm 8.1$ & $5315-5205$ & $5145-4985$ \\
\hline
\end{tabular}




\begin{tabular}{|c|c|c|c|c|c|c|c|c|c|c|c|c|c|c|}
\hline OxA-27560 & Sample 15, bone, Homo sapiens left femur & 620 & 14.13 & 2.3 & $43.2-20.3$ & 10.7 & - & 3.2 & $6178 \pm 29$ & $5220-5040$ & $16.7 \pm 9.5$ & - & $5220-5050$ & $5100-4935$ \\
\hline OxA-27584 & $\begin{array}{l}\text { from Burial } 134 \text {, trench } 33 \mathrm{~B} \text {, northern area } \\
\text { of cemetery. Male, } c .50 \text {. Right burial with } \\
\text { slightly flexed lower limbs. No grave goods. } \\
\text { Discrete glued repair at break away from } \\
\text { sample location. }\end{array}$ & 620 & 14.13 & 2.3 & $42.8-20.2$ & 10.0 & - & 3.1 & $\begin{array}{l}\left(\mathrm{T}^{\prime}=0.9\right. \\
\mathrm{T}^{\prime} 5 \%=3.8 \\
\mathrm{df}=1)\end{array}$ & & $15.0 \pm 9.0$ & - & & \\
\hline OxA-27561 & $\begin{array}{l}\text { Sample 12, bone, Homo sapiens right femur } \\
\text { from Burial 109, trench } 41 \text {, southern area of } \\
\text { cemetery. Female, estimated }<40 \text { for this } \\
\text { project. Left burial with slightly flexed } \\
\text { lower limbs containing a chisel. Discrete } \\
\text { glued repair at break away from sample } \\
\text { location. }\end{array}$ & 610 & 8.6 & 1.4 & $44.3-21.0$ & 10.6 & - & 3.3 & $6195 \pm 37$ & $5295-5040$ & $14.6 \pm 8.7$ & - & $5250-5040$ & $5115-4940$ \\
\hline OxA-27563 & Sample 29, bone, Homo sapiens humeral & 970 & 18 & 1.9 & $42.4-20.1$ & 10.1 & - & 3.2 & $6114 \pm 26$ & $5210-4945$ & $16.5 \pm 9.3$ & - & $5210-4990$ & $5060-4925$ \\
\hline OxA-27586 & $\begin{array}{l}\text { head from Burial } 321 \text {, trench } 98 \text {, southern } \\
\text { area of cemetery. Female, estimated } 55-60 \\
\text { for this project. Right-extended burial. No } \\
\text { grave goods. }\end{array}$ & 970 & 18 & 1.9 & $43.2-20.3$ & 10.1 & - & 3.2 & $\begin{array}{l}\left(\mathrm{T}^{\prime}=1.5\right. \\
\left.\mathrm{T}^{\prime} 5 \%=3.8\right)\end{array}$ & & $16.8 \pm 9.6$ & - & & \\
\hline OxA-27559 & Sample 11, bone, Homo sapiens left tibia & 630 & 11.22 & 1.8 & $42.7-20.2$ & 10.0 & - & 3.2 & $6163 \pm 28$ & $5215-5030$ & $14.8 \pm 9.1$ & - & $5220-5040$ & $5085-4930$ \\
\hline OxA-27583 & $\begin{array}{l}\text { from Burial 101, trench } 39 \mathrm{~A} \text {, northern area } \\
\text { of cemetery. Female, } 25-30 \text {. Supine } \\
\text { extended burial containing bone needle } \\
\text { with figurine and shell (Spondylus and } \\
\text { Dentalium) and malachite beads (tubular, } \\
\text { circular), and a flint fragment. Discrete } \\
\text { glued repair at break away from sample } \\
\text { location. }\end{array}$ & 630 & 11.22 & 1.8 & $43.5-20.2$ & 9.8 & - & 3.2 & $\begin{array}{l}\left(\mathrm{T}^{\prime}=0.0\right. \\
\mathrm{T}^{\prime} 5 \%=3.8 \\
\mathrm{df}=1)\end{array}$ & & $14.0 \pm 8.8$ & - & & \\
\hline OxA-27585 & Sample 21, bone, Homo sapiens left & 640 & 11.2 & 1.7 & $39.4-20.2$ & 10.0 & - & 3.2 & OxA-27585 & OxA-27430: & $14.7 \pm 8.7$ & - & $5215-5000$ & $5070-4935$ \\
\hline OxA-27562 & humerus from Burial 193, trench 86B, & 640 & 11.2 & 1.7 & $39-20.5$ & 9.9 & 0.8 & 3.2 & $5985 \pm 45$ & $5210-4960$ & $14.1 \pm 8.8$ & - & & \\
\hline OxA-27430 & $\begin{array}{l}\text { southern area of cemetery. Female, } \\
\text { estimated as } 30 \text { for this project. Left- } \\
\text { extended burial. No grave goods. Discrete } \\
\text { glued repair at break away from sample } \\
\text { location. } \\
\text { See main text. }\end{array}$ & 890 & 18.7 & 2.1 & $\begin{array}{ll}46 & -20.2\end{array}$ & 9.8 & & & $\begin{array}{l}\text { OxA-27562 } \\
5983 \pm 36 \\
\text { OxA-27430 } \\
6122 \pm 33\end{array}$ & & $14.4 \pm 8.7$ & $7.1 \pm 6.2$ & & \\
\hline OxA-27620 & $\begin{array}{l}\text { Sample 22, bone, Homo sapiens left femur } \\
\text { from Burial 194, trench } 86 \mathrm{~B} \text {, southern area } \\
\text { of cemetery. Female, 25-30. Extended } \\
\text { burial containing shell bi-lobed beads. }\end{array}$ & 870 & 33.8 & 3.9 & $\begin{array}{ll}43 & -20.2\end{array}$ & 9.2 & 4.9 & 3.3 & $6175 \pm 35$ & $5225-5010$ & $12.6 \pm 7.9$ & $14.8 \pm 9.8$ & $5225-5040$ & $5100-4930$ \\
\hline OxA-27630 & $\begin{array}{l}\text { Sample 27, bone, Homo sapiens neonate } \\
\text { left femur from Burial } 303 \text {, trench } 28 \text {, } \\
\text { northern area of cemetery. Grave } \\
\text { contained adult female, } 25-30 \text {, in left- } \\
\text { extended burial, with foetus } c .40 \text { weeks at } \\
\text { death, and Spondylus beads (tubular, } \\
\text { barrel-shaped). }\end{array}$ & 680 & 31 & 4.6 & $46.2-18.7$ & 11.2 & 3.2 & 3.2 & $6117 \pm 34$ & $5210-4950$ & $20.7 \pm 11.2$ & $14.8 \pm 10.1$ & $5215-4995$ & $5065-4920$ \\
\hline
\end{tabular}




\begin{tabular}{|c|c|c|c|c|c|c|c|c|c|c|c|c|c|c|c|}
\hline Lab code & Sample number and details & $\begin{array}{l}\text { Used } \\
(\mathrm{mg})\end{array}$ & $\begin{array}{l}\text { Yield } \\
(\mathrm{mg})\end{array}$ & $\begin{array}{l}\% \\
\text { yield }\end{array}$ & $\% \mathrm{C}$ & $\delta^{13} \mathrm{C}$ & $\delta^{15} \mathrm{~N}$ & $\delta^{34} \mathrm{~S}$ & $\begin{array}{l}\mathrm{C}: \mathrm{N} \\
\text { atomic } \\
\text { ratio }\end{array}$ & $\begin{array}{l}\text { Result } \\
\text { (BP) }\end{array}$ & $\begin{array}{l}\text { Calibrated } \\
{ }^{14} \mathrm{C} \text { date }(95 \% \\
\text { confidence; } \mathrm{BC})\end{array}$ & $\begin{array}{l}\text { FRUITS \% } \\
\text { estimated } \\
\text { freshwater fish } \\
\text { dietary } \\
\text { contribution } \\
\text { (model 1: C, N) }\end{array}$ & $\begin{array}{l}\text { FRUITS \% } \\
\text { estimated } \\
\text { freshwater fish } \\
\text { dietary } \\
\text { contribution } \\
\text { (model 2: C, N, S) }\end{array}$ & $\begin{array}{l}\text { Terrestrial- } \\
\text { no correction-- } \\
\text { preferred posterior } \\
\text { density estimate } \\
(95 \% \text { probability; } \\
\text { cal BC) }\end{array}$ & $\begin{array}{l}\text { Offset-correction } \\
\text { using FRUITS } \\
\text { model 1 - posterior } \\
\text { density estimate } \\
(95 \% \\
\text { probability; cal BC) }\end{array}$ \\
\hline OxA-27659 & $\begin{array}{l}\text { Sample 2, bone, Homo sapiens left femur } \\
\text { from Burial 12, trench 5, northern area of } \\
\text { cemetery. Female, 45-50. Right burial } \\
\text { with slightly flexed lower limbs. No grave } \\
\text { goods. }\end{array}$ & 530 & 17.04 & 3.2 & 40.2 & -19.8 & 9.4 & - & 3.2 & $6256 \pm 34$ & $5320-5075$ & $13.1 \pm 8.4$ & - & $5305-5075$ & $5135-4965$ \\
\hline OxA-28281 & $\begin{array}{l}\text { Sample 9, bone, Homo sapiens lower } \\
\text { mandible, horizontal right ram from } \\
\text { Burial } 62 \text {, northern area of cemetery, } \\
\text { trench } 25 \text {. Grave contained male } 25-35 \\
\text { for this project, right flexed burial. No } \\
\text { grave goods. Seems to overlap partially } \\
\text { grave } 61 \text {. }\end{array}$ & 1030 & 19.4 & 1.9 & 43.4 & -20.8 & 9.9 & 4.6 & 3.4 & $6206 \pm 31$ & $5295-5050$ & $14.0 \pm 8.5$ & $13.9 \pm 9.7$ & $5255-5055$ & $5115-4945$ \\
\hline OxA-28282 & $\begin{array}{l}\text { Sample } 10 \text {, bone, Homo sapiens upper } \\
\text { maxillary, left part. Burial } 97 \text { contained } \\
\text { male } 35 \text { years, unknown burial position, } \\
\text { northern area, trench } 37-38 \text {, with } \\
\text { microliths. }\end{array}$ & 1050 & 56.6 & 5.4 & 43.4 & -19.9 & 10.2 & 3.7 & 3.3 & $6172 \pm 32$ & $5220-5025$ & $15.6 \pm 9.0$ & $13.6 \pm 9.7$ & $5220-5040$ & $5095-4930$ \\
\hline $\begin{array}{l}\text { Poz-52598 } \\
\text { (Şerbănescu } \\
\text { 2015) }\end{array}$ & $\begin{array}{l}\text { Burial 284. Southern area, Homo sapiens, } \\
\text { supine extended burial. Grave included } \\
\text { one flint scraper, one bone ring, one bone } \\
\text { pendant. }\end{array}$ & - & - & - & - & - & - & - & - & $6095 \pm 35$ & $5210-4905$ & - & - & $5215-4980$ & $5060-4910$ \\
\hline \multicolumn{16}{|c|}{ Results from the settlement } \\
\hline OxA-27434 & $\begin{array}{l}\text { Sample 34, bone, Bos taurus right tibia } \\
\text { from Boian feature [102]. }\end{array}$ & 920 & 55.8 & 6.1 & 42.8 & -20.8 & 5.8 & 3.7 & 3.2 & $6099 \pm 34$ & $5210-4930$ & - & - & $5205-4930$ & - \\
\hline OxA-X-2511-19 & $\begin{array}{l}\text { Sample 32, bone, Cervus sp. phalanx I, } \\
\text { from Dudeşti pit [10]. }\end{array}$ & 810 & 7.3 & 0.9 & 44 & $-19.6^{*}$ & $5.5^{*}$ & 4.4 & & $6096 \pm 34$ & $5210-4910$ & - & - & $5205-4930$ & - \\
\hline OxA-27587 & Sample 31, bone, Bos taurus/primigenius & 450 & 10.2 & 2.3 & 42.2 & -19.9 & 7.2 & - & 3.2 & $6037 \pm 32$ & $5025-4840$ & - & - & $5050-4850$ & - \\
\hline OxA-27565 & Phalange II Dudeşti pit [10]. & 450 & 10.2 & 2.3 & 49.1 & -19.3 & 7.6 & - & 3.1 & $\begin{array}{l}\left(\mathrm{T}^{\prime}=0.1\right. \\
\mathrm{T}^{\prime} 5 \%=3.8 \\
\mathrm{df}=1)\end{array}$ & & - & - & & \\
\hline OxA-27564 & Sample 30, bone, Ovis/Capra ulna from & 400 & 17.4 & 4.4 & 42.6 & -18.6 & 7.5 & - & 3.2 & $6195 \pm 25$ & $5225-5050$ & - & - & $5220-5035$ & - \\
\hline OxA-27433 & Dudeşti pit $[10]$ & 400 & 17.4 & 4.4 & 42.4 & -18.7 & 7.6 & 2.9 & 3.2 & $\begin{array}{l}\left(\mathrm{T}^{\prime}=0.7\right. \\
\mathrm{T}^{\prime} 5 \%=3.8 \\
\mathrm{df}=1)\end{array}$ & & - & - & & \\
\hline
\end{tabular}


from Burial 29, trench 9, northern area of

cemetery. Male, estimated at $<30$ for this

project. Supine extended burial with grave

goods

P32947 Sample 8, bone, Homo sapiens left femur

from Burial 45 , trench $4 c$, northern area

of cemetery.

P32950 $\begin{aligned} & \text { Sample 16, bone, Homo sapiens right side } \\ & \text { of mandible from Burial 141, trench 34B, } \\ & \text { northern area of cemetery. }\end{aligned}$

P32950 $\begin{aligned} & \text { Sample 16, bone, Homo sapiens right side } \\ & \text { of mandible from Burial 141, trench 34B, } \\ & \text { northern area of cemetery. }\end{aligned}$

P32950 $\begin{aligned} & \text { Sample 16, bone, Homo sapiens right side } \\ & \text { of mandible from Burial 141, trench 34B } \\ & \text { northern area of cemetery. }\end{aligned}$

Sample 25, bone, Homo sapiens inferior

mandible from Burial 292, trench 24,

northern area of cemetery.

Sample failed due to low collagen yield

Sample failed due to low collagen yield

Sample failed due to low collagen yield

Sample failed due to low collagen yield 


\section{S Stratton et al.}

\section{MATERIALS AND METHODS}

\section{Sample Selection}

Samples for radiocarbon $\left({ }^{14} \mathrm{C}\right)$ dating were chosen to try to represent the full spatial extent of the cemetery from both the northern and southern areas, burials of different positions, ages, sexes and types of grave goods. Although the excavation uncovered a number of inter-cutting burials, the relevant burials were not present in the archive and could not be sampled. The human skeletal remains appeared in generally good condition. However, some past conservation techniques included the use of burning celluloid around broken bones to fix breaks. When possible, samples were from elements that had not been subject to these techniques, or as far from the area of burning as possible. Some samples of mandible, maxillae and teeth had been glued, and again sampling avoided these conserved areas.

We identified animal bone from the settlement both to explore the potential for a ${ }^{14} \mathrm{C}$ reservoir in human samples (e.g. Cook et al. 2001, 2009), and whether the cemetery and settlement phases were contemporary. The archive contained very limited faunal material from the settlement, and three samples were identified from a Dudeşti period pit [10], and one from a Boian period feature [102].

\section{Radiocarbon Measurement}

${ }^{14} \mathrm{C}$ measurements for this phase of work were made at the Oxford Radiocarbon Accelerator Unit (ORAU) using pretreatment, graphitization, and measurement by accelerator mass spectrometry (AMS) as outlined by Bronk Ramsey et al. (2002, 2004a, 2004b).

Stable carbon and nitrogen measurements were produced by isotope-ratio mass spectrometry at the ORAU (Bronk Ramsey et al. 2002, 2004a, 2004b). Sulphur isotope measurement was conducted at the Department of Anthropology, University of British Columbia, on excess collagen produced at ORAU according to methods outlined by Nehlich et al. (2010). Work along the Danube has highlighted the importance of aquatic resources to Mesolithic and Neolithic populations (Bonsall et al. 1997; Cook et al. 2001, 2002, 2009; Borić et al. 2004; Borić and Miracle 2004; Nehlich et al. 2010), while for the Copper Age cemetery at Varna, a low marine contribution in the diets of some individuals has been suggested (Higham et al. 2007:643; Higham et al. 2018). Hence in this regional context it remains important to ascertain the presence or absence of aquatic reservoirs that may affect ${ }^{14} \mathrm{C}$ dates. We therefore approached dietary reconstruction using two approaches of Bayesian modeling. The first applied a FRUITS Bayesian modeling approach to carbon and nitrogen stable isotopes only (Fernandes et al. 2014). The second estimated the proportion of freshwater fish contributions from stable carbon, nitrogen and sulphur isotopes using FRUITS Bayesian modeling (further details are available in the online supplementary materials). We conclude that the existence of a freshwater reservoir effect at Cernica is unlikely and no correction of the obtained ${ }^{14} \mathrm{C}$ measurements is required. However, because of the limited data for a freshwater reservoir effect in the Cernica Neolithic samples, our estimates for the chronology of the cemetery will necessarily remain somewhat provisional, and establishing a better dietary baseline must be a priority for future research in the region.

\section{Dietary Offsets for Radiocarbon Dating}

Quantification of marine or freshwater reservoir effects can be challenging (Hedges 2004). While extensive work has examined and mapped the variation in marine ${ }^{14} \mathrm{C}$ reservoirs 
(cf. Reimer et al. 2013; Sayle et al. 2014, 2016), freshwater reservoirs are more complicated. Approaches to identify diet-induced ${ }^{14} \mathrm{C}$ offsets have included the dating of pairs of wellassociated terrestrial animal bone and human bone (i.e. "perfect pairs") to calculate the aquatic reservoir (see Bonsall et al. 2015 for summary). This work has demonstrated variability in the actual ${ }^{14} \mathrm{C}$ reservoir effect between and even within species from single bodies of freshwater (Keaveney and Reimer 2012; Keaveney et al. 2015).

For Cernica, no pairs of faunal material and human skeletal remains from sealed contexts were available. We have therefore presented the modeled results firstly without a freshwater reservoir correction as our preferred model based on our interpretation of the FRUITS results described above and in the online supplementary materials. In order to examine what effect a possible freshwater dietary offset would have on our dating results, we also present alternative modeling results on the basis of a dietary mixing model, applying an aquatic reservoir estimate (545 \pm 70 ${ }^{14} \mathrm{C}$ yr) calculated for the Danube Gorges region by Bonsall et al. (2015:35) in IntCal13 with the offset proportional to the contribution of freshwater fish as indicated by the FRUITS results based on carbon and nitrogen values alone (model 1). We also have considered that the Danube Gorges reservoir may represent an overestimate of the potential aquatic reservoir effect at Cernica. The underlying geology of the Danube Gorges region is complex and in places is dominated by Cretaceous and Jurassic limestones as well as metamorphic and igneous rocks and flysch of different geological ages while loess of Pleistocene age covers the entire Romanian Plain (southern Romania) with a significant proportion of carbonates (Borić and Price 2013: Figure 1 and references therein). In addition, the Danube is a larger water body than the Colentina river system on which Cernica is located. The proportion of dissolved carbonates is therefore likely to be greater in the Danube Gorges, and the freshwater reservoir effect would consequently be greater.

\section{Calibration of ${ }^{14} \mathrm{C}$ dates}

Results of the dating are first presented as conventional ${ }^{14} \mathrm{C}$ ages (Stuiver and Polach 1977) and are calibrated using OxCal v4.2 (Bronk Ramsey 2009a). Calibrated ${ }^{14} \mathrm{C}$ date ranges quoted in Table 1 are calculated using the intercept method (Stuiver and Reimer 1986).

The effect of possible dietary offsets on the dating results have then been calibrated using the atmospheric IntCal13 (Reimer et al. 2013), and with the reservoir calculated for the Danube Gorges (cf. Bonsall et al. 2015; $545 \pm 70{ }^{14} \mathrm{C}$ yr) using a model reflecting the proportion of freshwater contributions shown in Table 1 and illustrated in Figures 7 and 8. A freshwater contribution $(12.7 \pm 9)$ derived from the sulphur, carbon and nitrogen average contribution calculated in the dietary reconstruction modeling (see online supplementary materials) has been applied to the result (Poz-52598) for which no stable carbon or nitrogen isotope data are extant.

\section{Bayesian Chronological Modeling}

A Bayesian analysis (Buck et al. 1996; Bronk Ramsey 2009a) has been applied to the results from Cernica using OxCal v4.2. The algorithms applied can be derived from the brackets and OxCal Command Query Language 2 (CQL2) keywords. The outputs from this analysis are quoted in italics. A model for the uncorrected results is shown in Figure 7. This is our preferred model and ranges from it are quoted in the text. The same model corrected for the freshwater, diet-derived offset described above is shown in Figure 8. Differences in the two model outputs are shown in Tables 1 and 2. 
Table 2 Posterior density estimates for parameters calculated in the models shown in Figures 7 and 8 .

\begin{tabular}{|c|c|c|c|c|}
\hline Parameter name & $\begin{array}{l}\text { Terrestrial model } \\
\text { Posterior density } \\
\text { estimate ( } 95 \% \\
\text { probability) }\end{array}$ & $\begin{array}{l}\text { Terrestrial model } \\
\text { Posterior density } \\
\text { estimate ( } 68 \% \\
\text { probability) }\end{array}$ & $\begin{array}{l}\text { Offset model } \\
\text { Posterior density } \\
\text { estimate ( } 95 \% \\
\text { probability) }\end{array}$ & $\begin{array}{l}\text { Offset model } \\
\text { Posterior density } \\
\text { estimate }(68 \% \\
\text { probability) }\end{array}$ \\
\hline Start Cernica cemetery (cal BC) & $5355-5220$ & $5295-5220$ & $5200-5000$ & $5100-5015$ \\
\hline End Cernica cemetery (cal BC) & $\begin{array}{l}5190-5080(28 \%) \text { or } \\
5070-4940(67 \%)\end{array}$ & $\begin{array}{l}5185-5150(13 \%) \text { or } \\
5045-4970(56 \%)\end{array}$ & $5040-4875$ & $5020-4935$ \\
\hline Duration cemetery (years) & $\begin{array}{c}30-155(27 \%) \text { or } \\
165-345(68 \%)\end{array}$ & $\begin{array}{l}45-85(13 \%) \text { or } \\
195-315(56 \%)\end{array}$ & $1-265$ & $1-130$ \\
\hline FirstNorth Cemetery (cal BC) & $5305-5210$ & $5270-5215$ & $5190-4995$ & $5085-5010$ \\
\hline FirstSouth Cemetery (cal BC) & $5330-5215$ & $5270-5220$ & $5170-4995$ & $5090-5015$ \\
\hline Start settlement (cal BC) & $5540-5015$ & $5240-5065$ & $5545-5015$ & $5240-5065$ \\
\hline End settlement ( cal BC) & $5045-4525$ & $4995-4830$ & $5045-4530$ & $4995-4830$ \\
\hline Duration settlement (years) & $30-305$ & $80-220$ & $35-305$ & $80-225$ \\
\hline
\end{tabular}

\section{RESULTS}

There are $31{ }^{14} \mathrm{C}$ results from the Cernica cemetery and the associated settlement (Table 1). These were produced on samples from 21 individuals from the cemetery ( 25 dates), and 4 faunal samples (6 dates) from 2 features (pit [10] and feature [102]) from the settlement. As noted above, we have very limited "informative" prior information (Bayliss et al. 2007) with which to constrain our data.

Three results (OxA-27585; -27562; -27430) on sample 21 are statistically inconsistent $\left(\mathrm{T}^{\prime}=10.2\right.$; $\mathrm{T}^{\prime} 5 \%=6.0 ; \mathrm{df}=2$; Ward and Wilson 1978; cf. Bronk Ramsey 2009b). OxA-27585 had a low target current and an offset in the $\delta^{13} \mathrm{C}$ values on the mass spectrometer and AMS. The low target current means that the standard error term is higher than usual, and the determination should be treated with caution. The autoduplicate ${ }^{3}$ OxA-27562 had a noticeably low $\% \mathrm{C}$ value on combustion. This is unusually low for collagen samples and may indicate a possible problem with the sample preparation for this AMS date. These results have not been included in the analysis (Figure 7), with only OxA-27430 included in the model. OxA-27630 was obtained on the remains of a neonate in Burial 303. The $\delta^{15} \mathrm{~N}$ of $11.2 \%$ is slightly elevated but not by much compared to other burials in the series and hence probably does not reflect a nursing signal.

Key posterior density estimates are given in Table 2 providing the comparison between the terrestrial (no correction applied) and offset models (correction applied based on FRUITS results) (see online supplementary materials). According to the preferred terrestrial model, use of the cemetery began in 5355-5220 cal BC $(95 \%$ probability; Terrestrial Start Cernica cemetery; Figure 7), probably in 5295-5220 cal BC (68\% probability). It appears that both areas of the cemetery were in use at the same time, with the north area first used in 5305-5210 $\mathrm{cal} \mathrm{BC}(95 \%$ probability; Terrestrial FirstNorthCemetery; Figure 7), probably in 5270-5215 cal BC (68\% probability). The south cemetery was first in use in 5330-5215 cal BC (95\% probability; Terrestrial FirstSouthCemetery; Figure 7), probably in 5270-5220 cal BC (68\% probability). Use of the cemetery ceased in 5190-5080 cal BC (28\% probability) or 5070-4940 (67\% probability; Terrestrial End Cernica cemetery; Figure 7), probably in 5185-5150 cal BC (13\% probability) or $5045-4970$ ( $56 \%$ probability). 


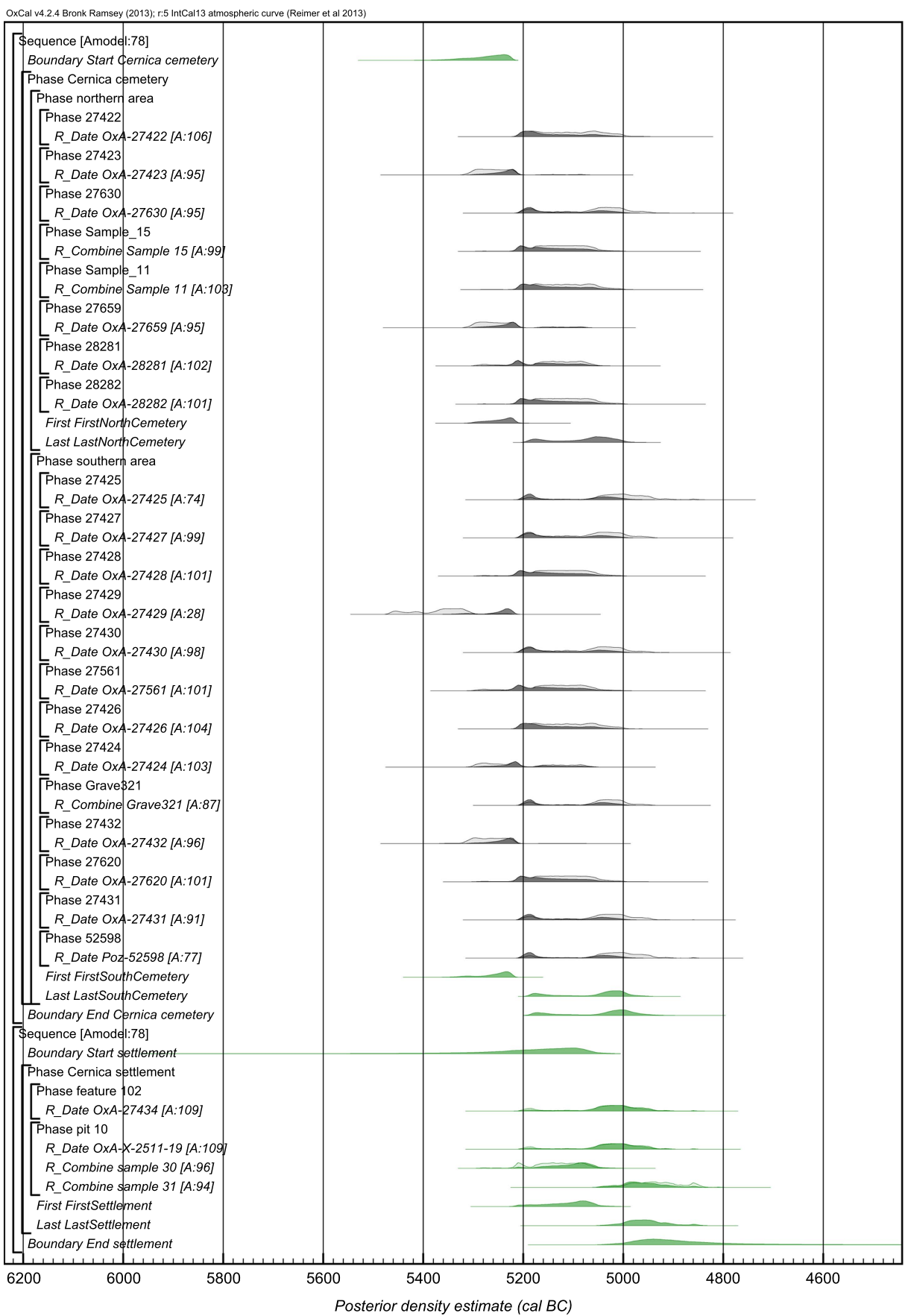

Figure $7{ }^{14} \mathrm{C}$ results from Cernica cemetery and settlement. These results have not been corrected for a diet-derived freshwater offset. For each ${ }^{14} \mathrm{C}$ result two distributions are plotted. In outline are the calibrated ${ }^{14} \mathrm{C}$ results; the green distributions are posterior density estimates, the outputs from the model using the terrestrial calibration curve. The OxCal CQL2 keywords and brackets define the model. 


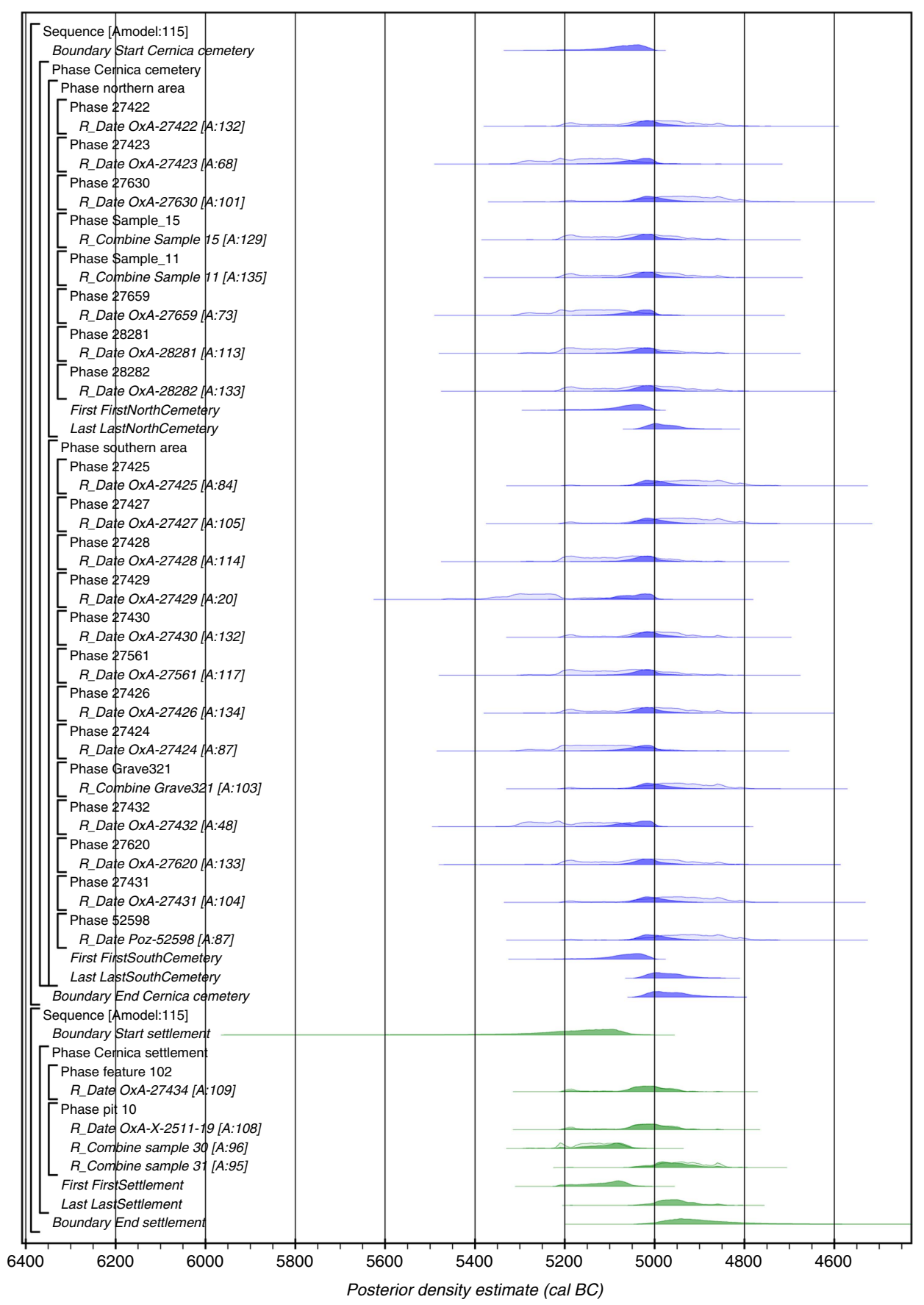

Figure $8{ }^{14} \mathrm{C}$ results from Cernica cemetery and settlement. These results have been corrected for a diet-derived freshwater offset as described in the text. For each ${ }^{14} \mathrm{C}$ result two distributions are plotted. The distributions in green have been calculated using the IntCal13 terrestrial curve; the distributions in blue have been calculated using a mix of calibration curves reflecting the estimated freshwater resource consumption and a local offset as described in the text. The OxCal CQL2 keywords and brackets define the model. 


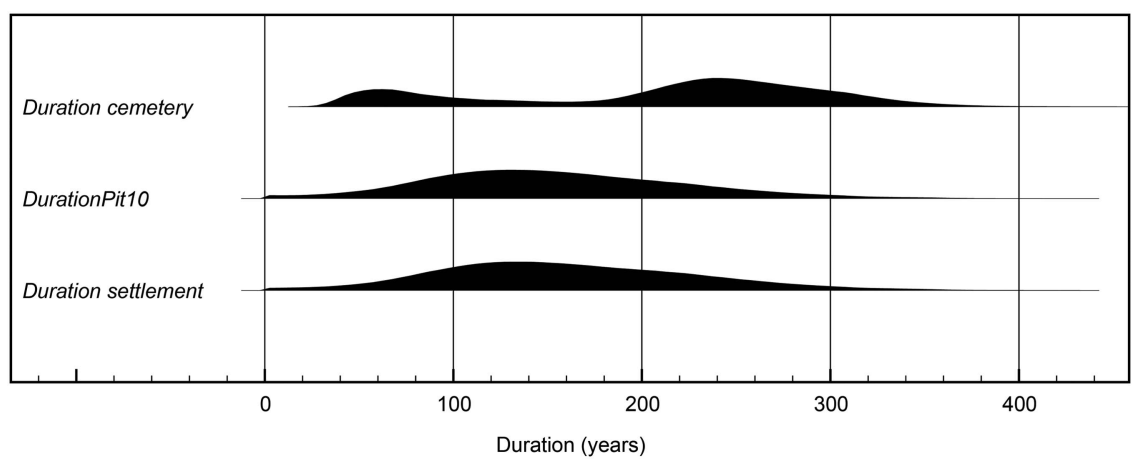

Figure 9 Posterior density estimates for the duration of different aspects of Cernica. The parent phases are respectively Cernica cemetery, pit 10, and settlement defined in the model in Figure 7.

If the freshwater reservoir offset is applied, the cemetery began in 5200-5000 cal BC $(95 \%$ probability; Offset Start Cernica cemetery; Figure 8), probably in 5100-5015 cal BC (68\% probability). It appears that both areas of the cemetery were in use at the same time, with the north area first used in 5190-4995 cal BC (95\% probability; Offset FirstNorthCemetery; Figure 8), probably in 5085-5010 cal BC (68\% probability). The south cemetery was first in use in 5190-4995 cal BC (95\% probability; Offset FirstSouthCemetery; Figure 8), probably in 5085-5010 cal BC (68\% probability). Use of the cemetery ceased in 5040-4875 cal BC (95\% probability; Offset End Cernica cemetery; Figure 8), probably in 5020-4935 cal BC (68\% probability).

The estimates here suggest that the cemetery was in use, according to the terrestrial model, for $30-155$ years (27\% probability) or 165-345 (68\% probability; Terrestrial Duration Cernica cemetery; Figure 9), probably for 45-85 years (13\% probability), or, according to the offset model for 1-265 years (95\% probability; Offset Duration Cernica cemetery; Figure 9), probably for $1-130$ years $(68 \%$ probability).

From a Boian feature [102] from the settlement, a cow bone was dated, while three bones - from a deer, cow, and sheep-were dated from Dudeşti pit [10]. The results from the Dudeşti pit (OxA-X-2511; OxA-27433; OxA-27564; OxA-27565; OxA-27587) are not statistically consistent $\left(\mathrm{T}^{\prime}=17.7 ; \mathrm{T}^{\prime} 5 \%=9.5 ; \mathrm{df}=4\right)$, with the results on sample 30 , a sheep/goat ulna (OxA-27564 and OxA-27433), probably older than the other results from the feature. The faunal samples from the settlement were all articulating or paired with other material from the same animal, and the inconsistency of the results from this feature is therefore difficult to explain. An interpretation is that the earliest results on sample 30 represents residual material, which along with other articulating elements from this individual, were redeposited in a feature during later activity.

The results from these features give some idea of the settlement activity, and we estimate that according to both terrestrial and offset models the settlement started in 5540-5015 cal BC (95\% probability; Start settlement; Figure 7), probably in 5240-5065 cal BC (68\% probability), and ended in 5045-4525 cal BC (95\% probability; End settlement; Figure 7), probably in 4995-4830 cal $B C(68 \%$ probability). The very limited sample size from the settlement means that these estimates might well not be representative of the full duration of activity. The currently available evidence is estimated to have occurred over a duration of 30-305 years (95\% probability; Duration settlement; Figure 9), probably over $80-225$ years (68\% probability). 
Interestingly the results from the Dudeşti period pit (OxA-X-2511; OxA-27565; OxA-27587) and the result from the Boian period feature $(\mathrm{OxA}-27434)$ are statistically consistent $\left(\mathrm{T}^{\prime}=2.4\right.$; $\mathrm{T}^{\prime} 5 \%=7.8 ; \mathrm{df}=3$ ) and could be of the same actual age. However, as we were only able to make one measurement on a Boian feature, we should be cautious about how representative this single measurement is of Boian period occupation.

From the available sample and according to both models, the start of activity associated with the settlement (Terrestrial Start settlement; Figure 7) overlaps with the current estimate for the start of use of the cemetery (Terrestrial Start cemetery; Figure 7).

Further work is required to explore the appropriateness of the Danube Gorges reservoir for the Cernica population. We think that given the similarity of the results on terrestrial herbivores, our model of the cemetery is unlikely to provide erroneously old estimates for burial. Also, there remains the potential that in the offset model we have applied too great a reservoir and that the cemetery might actually have been founded somewhat earlier-potentially more in keeping with the available dates for the settlement. For this reason, our preferred model is terrestrial with no reservoir offset applied. However, it is important to note that the difference in posterior density estimates between the two models does not greatly affect our overall conclusions.

\section{DISCUSSION}

\section{Chronological Implications for the Regional Context of the Lower Danube Basin}

Considering that the estimates for the start of the cemetery and settlement overlap and that the dated settlement features are dominantly assigned to the Dudeşti-Cernica phase based on the presence of typical pottery, we suggest that the cemetery, where diagnostic and sensitive chronological markers for a precise chronological attribution on the basis of material culture alone are often absent, started during this late Dudeşti phase and possibly continued into the early phase of the Boian culture, known as the Boian-Bolintineanu phase.

Previously, two other broadly contemporaneous cemetery sites from the Lower Danube area have been ${ }^{14} \mathrm{C}$ dated: Durankulak (Honch et al. 2013) and Varna I (Higham et al. 2007), both on the Black Sea coast. The dates for these cemeteries place them in the region's Late Neolithic and Early Copper Age, with Durankulak having two cultural phases: the Hamangia phase (contemporaneous with the Boian), and the Varna phase (a regional variant of the Gumelnitsa culture that takes its name from the Varna I cemetery type site). The results from Durankulak and Varna are shown together with our two models for Cernica in Figure 10.

When the posteriors for the estimate for the start of activity associated with the Cernica cemetery and the estimate for the start of activity associated with the Hamangia phase of cemetery activity from Durankulak are compared, the start of activity at Durankulak occurred after the start of activity at the Cernica cemetery. The Durankulak Hamangia phase most probably began in the second half of the 51 st century cal BC or the first half of the 50th century cal BC (68\% probability), while the Cernica cemetery most probably was first used from the second half of the 54th or in the 53rd century cal BC ( $68 \%$ probability). Use of the Cernica cemetery, however, probably ceased (Terrestrial End_cemetery) before the end of the Hamangia phase and the start of the Varna phase at Durankulak (StartDurankulakVarnaPhase; Figure 10). 


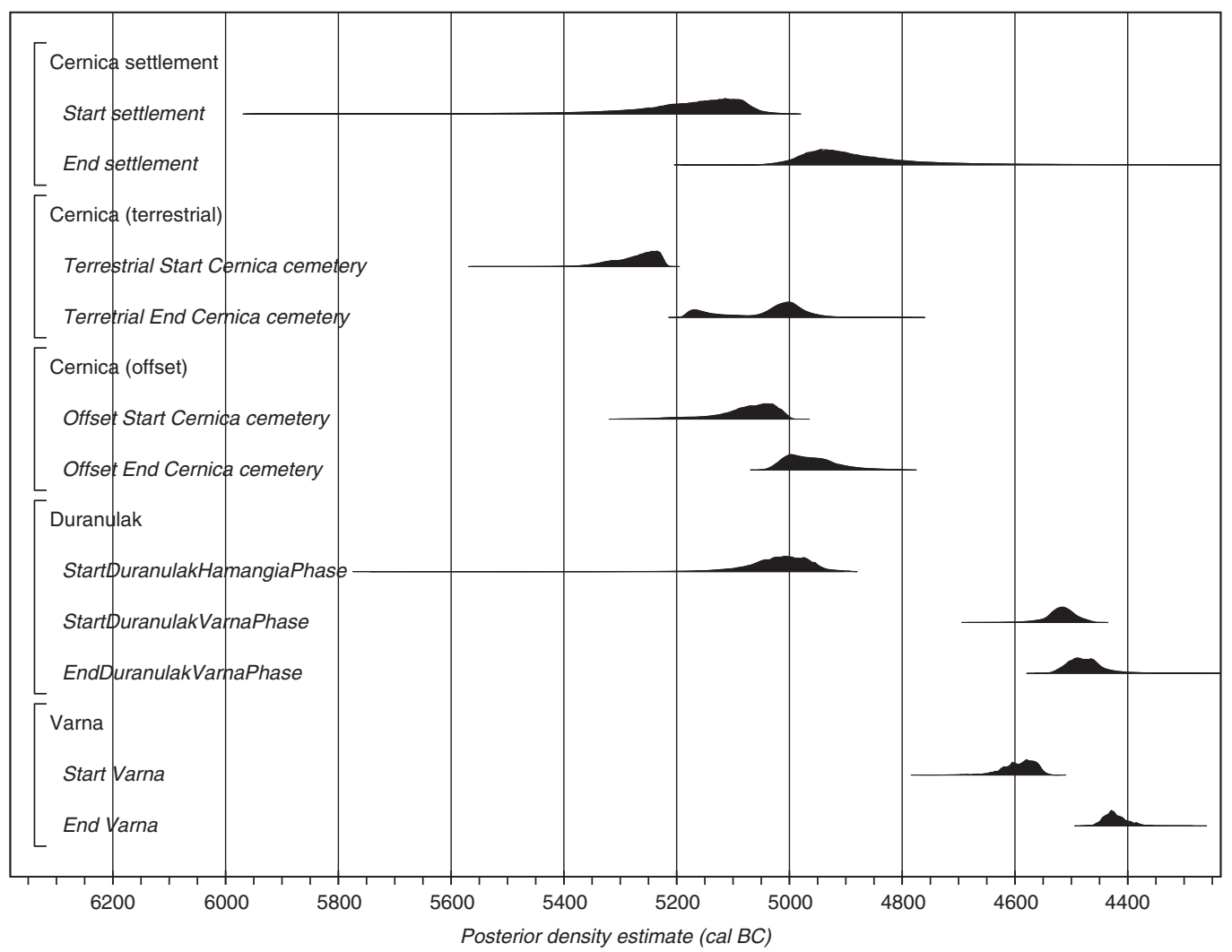

Figure 10 Key posterior density estimates from Cernica (as calculated in the models shown in Figures 7 and 8 ), Varna I, and Durankulak.

\section{Other Regional Traditions in Burial Rites?}

At Cernica, we have dated both the dominant extended supine inhumations (11 burials) as well as a range of variations of burials placed on either lateral right or left sides ( 9 burials). The chronological estimates for either of these two groupings are statistically indistinguishable and, for the moment, it is not possible to confirm earlier assumptions about the chronological distinction between these different body positions. In the latter group there are variations in the degree of flexion of lower limbs from those that are placed on either of the lateral sides as extended with no flexing (four dated burials), where only lower limbs were slightly flexed at the knees to those where femurs were flexed at the pelvis and legs at the knees. Hence different from many other burials in Neolithic southeast Europe, Cernica shows a strong tendency for extended burial position as the dominant norm even though some variations be noted.

Two different burial traditions can be identified in the Late Neolithic of Muntenia, northeast Bulgaria and Dobrudza, not necessarily following the borders of the culture groups traditionally defined on the basis of pottery styles. The first is characterised by flexed inhumations, the majority of which were found placed on their left sides, and oriented east-west. The second 


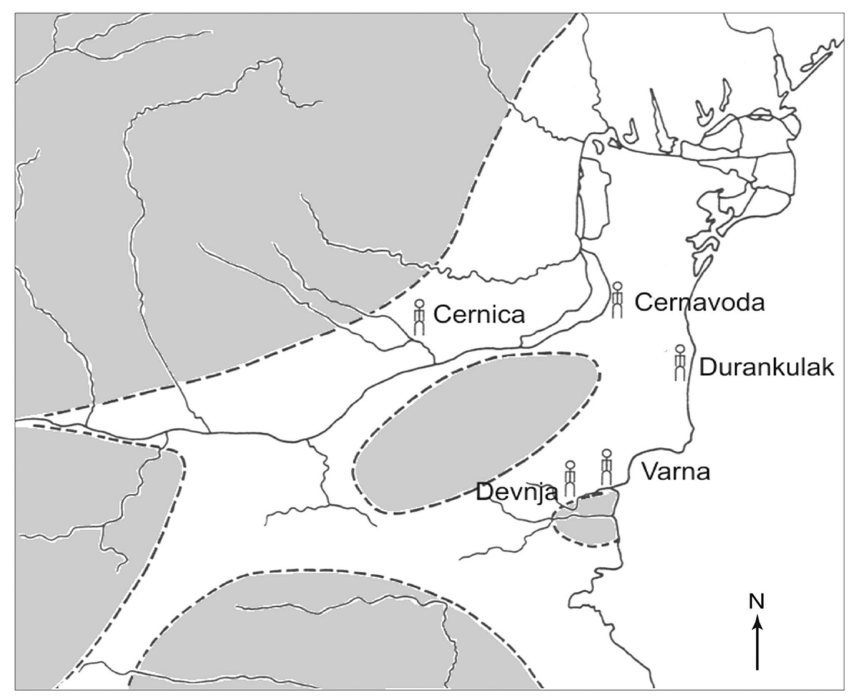

Figure 11 Map showing areas with Neolithic and Copper Age cemeteries containing extended supine burials as possible indications of Mesolithic mortuary rites in Muntenia, northeast Bulgaria and Dobrudza; shaded areas indicate zones of primary Neolithization in these regions (adapted after Lichter 2001:Figure 71).

(as at Cernica) or north-south (as in the majority of burials at the Hamangia culture cemetery of Durankulak). These elements, along with the presence of particular types of offerings or decorative items (such as red deer canines) in burials at Durankulak and Cernica, may suggest that regardless of the adoption of most of the common Neolithic materialities and practices (pottery, subsistence based on cultivation and domestic stock, and similar), there were areas in this part of the Balkans that might have adhered to much older Mesolithic rites, which included the use of perforated red deer canines as ornaments, the association of antler axes with male burials, and the placement of (wild) animal skulls to accompany the deceased (for a similar argument, see Todorova 2002:46-7).

Moreover, it has been noted that some of these new culture groups in the Dobrudza, Muntenia and the Black Sea coastal regions are the first Neolithic communities of their areas. The map (Figure 11) shows there are no overlaps between the distribution of extended supine burials during this later period and the presence of Early Neolithic communities in the region (cf. Lichter 2001:151-3). While the most recent research might have altered this pattern somewhat with the discovery of Early Neolithic sites around the area where Cernica is located, it still remains relevant for the region of Dobrudza. The only Mesolithic burials in the wider region of the eastern Balkans that were placed in extended supine positions, seen as a dominant Mesolithic burial rite for the whole of Europe (cf. Grünberg 2000), were in the Danube Gorges (e.g. Borić 2011, 2016). In addition, the flint inventories of Dudeşti, early Boian, early Hamangia and Usoe cultures may also suggest certain Mesolithic traits (Gatsov 1982; Păunescu 1988). While these instances suggest a degree of hybrid cultural identities created in the course of the Middle-Late Neolithic in this region, they possibly also suggest strong differences reflected in the belief systems of particular communities that might have had different origin myths: one aligning itself with Neolithic descent from the east and the other with Mesolithic descent from the north and west (Borić 2015). 
And yet, there remains the enigma regarding the invisibility of such "Mesolithic" traits in the archaeological record of this particular micro-region prior to the appearance of Cernica. While skeletal remains may not be in a sufficient state of preservation for an informative analysis of the characteristics of the population at Cernica solely based on osteological parameters, future aDNA analyses on this material could possibly provide indications about the genetic ancestry of the population that composes the cemetery, whether derived from hunter-gatherer or farming populations, or a mixture of the two. Recent aDNA work in reconstructing the genomic history of southeast Europe indicates that some degree of mixing between local forager population and farmers originating in northwest Anatolia took place precisely along the Danube River Valley. Many newly arrived Neolithic populations in the Balkans seem to have remained genetically separate from indigenous foragers for a long time (Mathieson et al. 2018). However, the phenomenon of the "resurgence" of hunter-gatherer ancestry, which was previously reported in the Middle Neolithic of central Europe and Iberia (Brandt et al. 2013; Haak et al. 2015; Mathieson et al. 2015), is now also reported for the Copper Age Balkans (Mathieson et al. 2018). This could mean that more intensive mixing between forager and farmer populations occurred in various areas of Europe once indigenous foragers became fully "Neolithic". However, we should not expect that knowing more about the genetic make-up of a population buried in this and other cemeteries will necessarily provide sufficient clues to the modalities and motivations of how particular cultural practices were being chosen, transformed and abandoned. Cultural traditions might have been adopted, modified or reinvented regardless of population continuities and discontinuities (cf. Wagner 1981).

\section{Emerging Cemeteries and Social Relations}

The emergence of the cemetery phenomenon, which this project has dated at Cernica to 53555215 cal BC (95\% probability; Start cemetery; Figure 7) as one of the earliest securely dated examples, hints at new ways of thinking, not simply about the treatment of the dead, but also the role of the dead in society and the creation of community identity. While previously, in the Early and Middle Neolithic, the remains of the dead were found primarily within settlements, this new practice of establishing extramural cemeteries created a permanent, separate and visible place for the dead. Extramural cemeteries with rows of burials can be seen as a particular innovation in mortuary rites that seem to have emerged in the Lower Danube area most probably in the late 54th or 53rd century cal BC at Cernica and in the second half of the 51st century cal BC or the first half of the 50th century cal BC at Durankulak. This change in burial practice may also be representative of broader social and perhaps also demographic changes, in which the need for creating a permanent place for the dead can be linked to claims over land, establishment of place and/or creation of particular communities (e.g., Chapman 1996, 2000). In this context, such a build-up of long genealogies depended on "the proper combination of ancestral corpses and ancestral land" (Bloch and Perry 1982:7).

The settlement area at Cernica was not fully excavated, and for the moment we cannot make any reliable estimate about settlement size. The burials in the cemetery present a roughly even number of adult males and females with age ranges from infants to individuals of 60 years old. Such a distribution may be thought indicative of the inclusion of a whole population in cemetery burial. However, children are certainly under-represented at a time of presumably high child mortality (Kogălniceanu 2008). They at least are not all being buried in the cemetery area, and it is possible that for whatever reason certain adults were not, either. After all, for the preceding period the majority of the population are not visible at all in the archaeological record. It is difficult to make any statement therefore on whether the cemetery burials at Cernica are exclusively from the adjacent settlement, or whether most individuals from the settlement 


\section{S Stratton et al.}

were buried there; we simply note the 378 burials, which at a rate of two or three burials a year might be a reasonable number for such a settlement size over the possible (though uncertain) period of use that is currently estimated at 30-155 years (27\% probability) or $165-345$ (68\% probability) according to the terrestrial model.

Our results do not indicate any spatial pattern of chronological development of the cemetery. It seems that both the northern and southern areas of the cemetery were in use simultaneously, and if this is the case then we may see the two separate burial clusters as representing a social segmentation, which could be different families, clans or other groupings. If these burial nuclei represent some kind of kinship grouping, it is possible that the outliers from the main group, that is, those that lie between the two clusters and those to east and west, had different allegiances and affiliations (Ursulescu and Kogălniceanu 2007).

So, what was the separation of the Cernica cemetery from the adjacent settlement all about for the collective that used it? Was it the first step in the process that John Chapman (1996) identifies in later, Early Copper Age, practices, of establishing large extramural cemeteries? That was seen as a move motivated by the desires or needs of social actors to break away from rigid arrangements, as suggested by settlement layouts, by creating a novel arena of social power, with its own logic and space in which it was possible to express alternative discourses of power and self-expression. Or, alternatively, were the layout and rules in the mortuary domain closely following social arrangements in the place of the living? Can we see in these emerging burial grounds in the eastern Balkans a novel social space that can be equated with corporate lineages, with a cemetery serving a wider region and not only nearby contemporaneous settlements? Moreover, who composed this collective and what social dynamics contributed to the spread of the cemetery and the formation of distinct burial rows? Do burial rows signify ties based on kinship? Finally, what social structure held this collective together in the recurrent use of the same cemetery ground?

The current state of the evidence does not allow us to answer most of these questions with any degree of certainty. Some clarification could be achieved by future strontium isotope and aDNA analyses of the available skeletal evidence, better understanding of the social dynamics and the chronology of the adjacent settlement as well as by providing comparable datasets within the wider region. Similarly, more AMS dates for various burial groupings and rows within the cemetery would allow for a finer-tuned chronology of what Chapman (2000) calls micro-traditions of mortuary rites.

On the face of the evidence, this cemetery primarily, if not exclusively, served the nearby settlement. The overall homogeneity of burial rites and the lack of status distinctions among the burials, apart from a few ornaments and some exotica, such as Spondylus, Glycymeris, Dentalium and copper or malachite would suggest elements of horizontal differentiation characteristic of a segmentary society with a largely egalitarian ethos. Funerals were, among other things, social and political events (Parker Pearson 1999), but at Cernica, the key development appears to have been the formalization of a burial area, and it was inclusion in that, rather than differentiation within it, that seems to have mattered. Nor does differentiation appear to be seen in the settlements of the period. The appearance of the body in the grave could have said subtle things about the deceased individual's personal identities through the inclusion of items which represented gender, age, descent group or social roles - the way personhood might have been constructed in this social and cultural milieu - but it seems that it was membership above all which was projected (Borić et al. 2013:53). On the other hand, in this particular instance, making a case for the argument that an alternative arena of social power was created away from 
the settlement would require a more competent understanding of the dynamics at the Cernica settlement itself and this can only be tested by future fieldwork.

\section{ACKNOWLEDGMENTS}

The dating reported here was made possible by NERC grant NF/2012/1/4, made to Alasdair Whittle on behalf of the Cardiff University PhD thesis of Susan Stratton (2017) and in collaboration with Raluca Kogălniceanu, Seren Griffiths and Dušan Borić. We are grateful to the Oxford Radiocarbon Accelerator Unit for their help.

\section{SUPPLEMENTARY MATERIAL}

To view supplementary material for this article, please visit https://doi.org/10.1017/ RDC.2018.34

\section{REFERENCES}

Bayliss A, Bronk Ramsey C, van der Plicht J, Whittle A. 2007. Bradshaw and Bayes: Towards a timetable for the Neolithic. Cambridge Archaeological Journal 17.1, supplement:p 1-28.

Berciu D, Morintz S. 1957. Şantierul arheologic Cernavoda (reg. Constanţa, r. Medgidia). Materiale 3:83-92.

Berciu D, Morintz S. 1959. Săpăturile de la Cernavoda (reg. Constanţa, r. Medgidia). Materiale 5:99-106.

Berciu D, Morintz S, Roman P. 1959. Săpăturile de la Cernavoda (reg. Constanţa, r. Medgidia). Materiale 6:95-105.

Berciu D, Morintz S, Ionescu M, Roman P. 1961. Şantierul arheologic Cernavoda. Materiale 7:49-55.

Bloch M, Parry J. 1982. Introduction: death and the regeneration of life. In: Bloch M, Parry J, editors. Death and the Regeneration of Life. Cambridge: Cambridge University Press. p. 1-44.

Bojadzhiev J. 2001. The intra muros burial practice during the Neolithic and Chalcolithic in the Bulgarian lands: Custom or exception? Archeologija (Sofia) 42/3-4:16-24.

Bonsall C. 2007. When was the Neolithic transition in the Iron Gates? In: Spataro M, Biagi P, editors. $A$ short walk through the Balkans: The first farmers of the Carpathian Basin and adjacent regions. Trieste: Società per la prehistoria e protostoria della regiona Friuli-Venezia Giulia. p. 53-66.

Bonsall C, Rosemary L, McSweeney K, Carolina S, Douglass H, Boroneanţ V, Bartosiewicz L, Robert P, Chapman J. 1997. Mesolithic and Early Neolithic in the Iron Gates: A palaeodietary perspective. Journal of European Archaeology 5:50-92.

Bonsall C, Vasić R, Boroneant A, Roksandic M, Soficaru A, McSweeney K, Evatt A, Aguraiuja Ü, Pickard C, Dimitrijević V, Higham T, Hamilton D, Cook GT. 2015. New AMS ${ }^{14} \mathrm{C}$ for human remains from stone age sites in the Iron Gates reach of the Danube, southeast Europe. Radiocarbon 57(1):33-46.

Borić D. 2009. Absolute dating of metallurgical innovations in the Vinča culture of the Balkans. In: Kienlin TK, Roberts BW, editors. Metals and Societies. Studies in Honour of Barbara S. Ottaway
(Universitätsforschungen zur prähistorischen Archäologie). Bonn: Habelt. p 191-245.

Borić D. 2011. Adaptations and transformations of the Danube Gorges foragers (c. 13,000-5500 cal BC): An overview. In: Krauß R, editor. Beginnings-new research in the appearance of the Neolithic between Northwest Anatolia and the Carpathian Basin. Papers of the International Workshop 8th-9th April 2009, Istanbul. Rahden: Marie Leidorf. p 157-203.

Borić D. 2015. Mortuary practices, bodies and persons in the Neolithic and Early-Middle Copper Age of southeast Europe. In: Fowler C, Harding J, Hofmann D, editors. The Oxford Handbook of Neolithic Europe. Oxford: Oxford University Press. p 927-57.

Borić D. 2016. Deathways at Lepenski Vir: Patterns in Mortuary Practice. Belgrade: Serbian Archaeological Society.

Borić D, Grupe G, Peters J, Mikić Ž. 2004. Is the Mesolithic-Neolithic subsistence dichotomy real? New stable isotope evidence from the Danube Gorges. European Journal of Archaeology 7(3):221-48.

Borić D, Harris OJT, Miracle P, Robb J. 2013. The limits of the body. In: Robb J, Harris OJT, editors. The Body in History: Europe from the Palaeolithic to the Future. Cambridge: Cambridge University Press. p 32-63.

Borić D, Miracle P. 2004. Mesolithic and Neolithic (dis) continuities in the Danube Gorges: New AMS dates from Padina and Hajdučka Vodenica (Serbia). Oxford Journal of Archaeology 23(4):341-71.

Borić D, Price TD. 2013. Strontium isotopes document greater human mobility at the start of the Balkan Neolithic. Proceedings of the National Academy of Sciences 110(9):3298-303.

Brandt G, Haak W, Adler CJ, Roth C, SzécsényiNagy A, Karimnia S, Möller-Rieker S, Meller H, Ganslmeier R, Friederich S, Dresely V, Nicklisch N, Pickrell J, Sirocko F, Reich D, Cooper A, Alt KW. 2013. Ancient DNA reveals key stages in the formation of central European mitochondrial genetic diversity. Science 342:257-61. 
Bronk Ramsey C. 2009a. Bayesian analysis of radiocarbon dates. Radiocarbon 51(1):337-60.

Bronk Ramsey C. 2009b. Dealing with outliers and offsets in radiocarbon dating. Radiocarbon 51(3): $1023-45$.

Bronk Ramsey C, Higham T, Bowles A, Hedges R. 2004a. Improvements to the pre-treatment of bone at Oxford. Radiocarbon 46(1):155-63.

Bronk Ramsey C, Higham T, Leach P. 2004b. Towards high precision AMS: progress and limitations. Radiocarbon 46(1):17-24.

Bronk Ramsey C, Higham T, Owen C, Pike A, Hedges R. 2002. Radiocarbon dates from the Oxford AMS system: Archaeometry datelist 31. Archaeometry 44(3), supplement 1:40-90.

Buck CE, Cavanagh WG, Litton CD. 1996. Bayesian Approach to Interpreting Archaeological Data. Chichester: Wiley.

Cantacuzino Gh, Morintz S. 1968. Descoperirile arheologice de la Cernica şi importanţa lor pentru cunoaşterea vechilor culturi din ţara noastră. Bucureşti 6:7-26.

Cantacuzino Gh, Morintz S. 1963. Die jungsteinzeitliche Funde in Cernica. Dacia 7: 27-91.

Chapman J. 1996. Enchainment, commodification, and gender in the Balkan Copper Age. Journal of European Archaeology 4:203-42.

Chapman J. 2000. Tensions at Funerals: MicroTradition Analysis in Later Hungarian Prehistory. Budapest: Archaeolingua.

Ciocănel (Vintilă) C-M. 2015. Neo-eneoliticul în zona oraşului Bucureşti şi judeţul Ilfov. Culturile Dudeşti, Boian, Gumelniţa. Unpublished $\mathrm{PhD}$ thesis, "Vasile Pârvan" Institute of Archaeology, Bucharest.

Comşa E. 1974a. Istoria Comunităţilor Culturii Boian. Bucharest: Editura Academei Române.

Comşa E. 1974b. Die Bestattungssitten im rumanischen Neolithikum. Jahresschrift fur mitteldeutsche Vorgeschichte 58:113-56.

Comşa E. 1975. Nouvelles données relatives à la phase Bolintineanu de la culture Boïan (à la lumière des fouilles de l'agglomération de Cernica). Dacia 19:19-26.

Comşa E. 1992. Despre datarea necropolei neolitice de la Cernica. Cercetări Arheologice în Bucureşti 4:31-6.

Comşa E, Cantacuzino G. 2001. Necropola Neolitică de la Cernica. Bucharest: Editura Academei Române.

Cook GT, Bonsall C, Hedges R, McSweeney K, Boroneanţ A, Bartosiewicz L, Pettitt PB. 2002. Problems of dating human bones from the Iron Gates. Antiquity 76:77-85.

Cook GT, Bonsall C, Hedges REM, McSweeney K, Boroneanţ A, Pettitt PB. 2001. A freshwater dietderived $14 \mathrm{C}$ reservoir effect at the Stone Age sites in the Iron Gates gorge. Radiocarbon 43(2A):453-60.

Cook GT, Bonsall C, Pickard C, McSweeney K, Bartosiewicz L, Boroneanţ A. 2009. The Mesolithic-Neolithic transition in the Iron Gates, southeast Europe: calibration and dietary issues. In: Crombé P, Van Strydonck M, Sergant J, Boudin M, Bats M, editors. Chronology and Evolution within the Mesolithic of North-West Europe. Newcastle upon Tyne: Cambridge Scholars Publishing. p 497-515.

Fernandes R, Millard A, Brabec M, Nadeau M-J, Grootes P. 2014. Food reconstruction using isotopic transferred signals (FRUITS): A Bayesian model for diet reconstruction. PLoS One 9:1-9.

Gatsov I. 1982. The Archaeological cultures at the Late Pleistocene and Early Holocene in the Western Black sea region and their significance for the formation of the Neolithic flint industries. In: Kozłowski JK, editors. Origin of the chipped stone industries of the early farming cultures in the Balkans. Warszawa-Krakow: Uniw. Jagiellońskiego. p 111-31.

Grünberg JM. 2000. Mesolithische Bestattungen in Europa. Ein Beitrag zur vergleichenden Gräberkunde. Auswertung und Katalog. Rahden/ Westf.: Verlag Marie L. Leidorf.

Haak W, Lazaridis I, Patterson N, Rohland N, Mallick S, Llamas B, Brandt G, Nordenfelt S, Harney E, Stewardson E, Fu Q, Mittnik A, Bánffy E, Economou C, Francken M, Friederich S, Pena RG, Hallgren F, Khartanovich V, Khokhlov A, Kunst M, Kuznetsov P, Meller H, Mochalov O, Moiseyev V, Nicklisch N, Pichler SL, Risch R, Guerra MAR, Roth C, Szécsényi-Nagy A, Wahl J, Meyer M, Krause J, Brown D, Anthony D, Cooper A, Alt KW, Reich D Massive migration from the steppe was a source for Indo-European languages in Europe. Nature 522(7555):207-11.

Hedges REM. 2004. Isotopes and red herrings: comments on Milner et al. and Lidén et al. Antiquity 78:34-7.

Higham T, Chapman J, Slavchev V, Gaydarska B, Honch N, Yordanov Y, Dimitrova B. 2007. New perspectives on the Varna cemetery (Bulgaria). AMS dates and social implications. Antiquity 81:640-54.

Higham T, Slavchev V, Gaydarska B, Chapman J. 2018. AMS dating of the Late Copper Age cemetery, Bulgaria. Radiocarbon. DOI:10.1017/ RDC.2018.9.

Honch NV, Higham T, Chapman JC, Gaydarska B, Todorova H, Slavchev V, Dimitrova B. 2013. West Pontic diets: a scientific framework for understanding the Durankulak and Varna I cemeteries, Bulgaria. Interdisciplinaria Archaeologica, Natural Sciences in Archaeology 4:147-62.

Keaveney EM, Reimer PJ. 2012. Understanding the variability in freshwater radiocarbon reservoir offsets: A cautionary tale. Journal of Archaeological Science 39:1306-16.

Keaveney EM, Reimer PJ, Foy RH. 2015. Young, old and weathered carbon: using radiocarbon and stable isotopes to identify carbon sources in an alkaline, humic lake. Radiocarbon 57(3): 407-23. 
Kogălniceanu R. 2005. Utilizarea testului $\chi^{2}$ în arheologie. Studiu de caz - necropola neolitică de la Cernica. Arheologia Moldovei 28:265-302.

Kogălniceanu R. 2008. Child burials in intramural and extramural contexts from the Neolithic and Chalcolithic of Romania: The problem of "inside" and "outside". In: Bacvarov K, editor. Babies Reborn: Infant/Child Burials in Pre- and Protohistory (BAR Int. Ser. 1832). Oxford: Archaeopress. p 101-11.

Kogălniceanu R. 2009. Primele necropole din neoliticul şi eneoliticul României [unpublished $\mathrm{PhD}$ thesis]. Universitatea Alexandru Ioan Cuza, Iaşi.

Kogălniceanu R. 2012. Human remains from the Mesolithic to the Chalcolithic period in southern Romania. An update on the discoveries. Archaeologia Bulgarica 16(3):1-46.

Lazăr C. editor. 2012. The Catalogue of the Neolithic and Eneolithic Funerary Findings from Romania. București-Târgovişte: Cetatea de Scaun.

Lichter C. 2001. Untersuchungen zu den Bestattungssitten des südosteuropaischen Neolithikums und Chalkolithikums. Mainz: von Zabern.

Mărgărit M, Vintilă C-M. 2015. New information from old collections. Reevaluation of personal adornments made of hard animal materials from the necropolis of Cernica. Studii de Preistorie 12:81-115.

Mathieson I, et al. 2015. Genome-wide patterns of selection in 230 ancient Eurasians. Nature 528:499-503.

Mathieson I, et al. 2018. The genomic history of southeastern Europe. Nature 555:197-203.

Morintz S, Berciu D, Diaconu P. 1955. Şantierul arheologic Cernavoda. Studii şi Cercetări de Istorie Veche 6:151-63.

Necrasov O, Critescu M, Botezatu D, Miu G. 1990. Cercetări paleoantropologice privitoare la populațiile de pe teritoriul României. Arheologia Moldovei 13:173-206.

Nehlich O, Borić D, Stefanović S, Richards M. 2010. Sulphur isotope evidence for freshwater fish consumption: a case study from the Danube Gorges, SE Europe. Journal of Archaeological Science 37:1131-9.

Parker Pearson M. 1999. The Archaeology of Death and Burial. Stroud: Sutton.

Păunescu A. 1988. Les industries lithiques du néolithique ancien de le Roumanie et quelques considérations sur l'inventaire lithique des cultures du néolithique moyen de cette contrée. Dacia 32:5-19.

Reimer P, Bard E, Bayliss A, Beck J, Blackwell P, Bronk Ramsey C, Grootes P, Guilderson T, Haflidason H, Hajdas I, Hatté C, Heaton T, Hoffmann D, Hogg A, Hughen K, Kaiser K, Kromer B, Manning S, Niu M, Reimer R, Richards D, Scott E, Southon J, Staff R, Turney C, van der Plicht J. 2013. IntCal13 and Marine13 radiocarbon age calibration curves $0-50,000$ years cal BP. Radiocarbon 55(4):1869-87.
Rosenstock E, Scharl S, Schier W. 2016. Ex oriente lux? - Ein Diskussionsbeitrag zur Stellung der frühen Kupfermetallurgie Südosteuropas. In: Bartelheim M, Horejs B, Krauß R, editors. Von Baden bis Troia: Ressourcennutzung, Metallurgie and Wissenstransfer. Eine Jubiläumsschrift für Ernst Pernicka. Rahden-Westf.: Marie Leidorf GmbH. p 59-122.

Sayle K, Cook GT, Ascough PL, Gestsdóttir H, Hamilton WD, McGovern TH. 2014. Utilization of $\delta^{13} \mathrm{C}, \delta^{15} \mathrm{~N}$, and $\delta^{34} \mathrm{~S}$ analyses to understand ${ }^{14} \mathrm{C}$-dating anomalies within a Late Viking Age community in northeast Iceland. Radiocarbon 56 (2):811-21, doi: 10.2458/56.17770.

Sayle KL, Hamilton WD, Gestsdóttir H, Cook GT. 2016. Modelling Lake Mývatn's freshwater reservoir effect: Utilisation of the statistical program FRUITS to assist in the re-interpretation of radiocarbon dates from a cemetery at Hofstaðir, north-east Iceland. Quaternary Geochronology. Available at http://eprints.gla.ac.uk/121200/.

Schuster C, Kogălniceanu R, Morintz A. 2008. The Living and the Dead: an Analysis of the Relationship between the Two Worlds during Prehistory at the Lower Danube. Târgovişte: Editura Cetatea de Scaun.

Şerbănescu D. 1999. Necropola neolitică de la Popeşti, com. Vasilaţi, jud. Călăraşi. In: Neagu M, editor. Civilizaţia Boian pe teritoriul Romaniei. Călăraşi. p 14-6.

Şerbănescu D. 2002. Observaţii preliminare asupra necropolei neolithice de la Sultana, judeţul Călăraşi. Cultură şi Civilizaţie la Dunărea de Jos, Călăraşi 19:69-86.

Şerbănescu D. 2015. Consideraţii privind datarea necropolei neolitice de la Cernica, judeţul Ilfov. In: Schuster C, Tulugea C, Terteci C, editors. Volum dedicat profesorului PETRE I. ROMAN la cea de-a 80-a aniversare (Buridava XII/1, Symposia Thracologica X). Râmnicu Vâlcea. p 116-20.

Şerbănescu D, Soficaru A. 2006. Sultana, com. Mănăstirea, jud. Călăraşi, Punct: Valea Orbului. In: Cronica Cercetărilor Arheologice din Romania. Campania 2005. A XL-a Sesiune Naţională de Rapoarte Arheologice, Constanţa, 31 mai-3 iunie 2006. Bucureşti. p 343-7.

Şerbănescu D, Cristache S. 2011. Curăteşti, com. Frăsinet, jud. Călăraşi. Punct: Biserica Veche. In: Cronica Cercetărilor Arheologice. Campania 2010. A XLV-a Sesiune Naţională de Rapoarte Arheologice, Sibiu, 26-29 mai 2011. Sibiu. 43.

Şerbănescu D, Comşa A, Mecu L. 2007. Sultana, com. Mănăstirea, jud. Călăraşi. Punct: Valea Orbului. Cronica Cercetărilor Arheologice din România. Campania 2006. A XLI-a Sesiune Naţională de Rapoarte Arheologice, Tulcea, 29 mai-1 iunie 2007. Bucharest: cIMeC. p 351-2.

Şerbănescu D, Nica T, Comşa A, Laurenţiu M. 2008. Sultana, com. Mănăstirea, jud. Călăraşi. Punct: Valea Orbului, Cronica Cercetărilor Arheologice din România. Campania 2007. A XLII-a Sesiune 
Națională de Rapoarte Arheologice, Iaşi, 14-17 mai. Bucharest: cIMeC. p 294-5.

Stratton S. 2017. Burial and Identity in the Late Neolithic and Copper Age of Southeast Europe [unpublished $\mathrm{PhD}$ dissertation]. Cardiff University.

Stuiver M, Polach HA. 1977. Discussion: reporting of ${ }^{14} \mathrm{C}$ data. Radiocarbon 19(3):355-63.

Stuiver M, Reimer PJ. 1986. A computer program for radiocarbon age calculation. Radiocarbon 28(2): 1022-30.

Todorova H, Vasilev V, Januševič Z, Kovačeva M, Vlev P. 1983. Ovčarovo. Sofija: Razkopki i Proučvanija VIII.
Todorova H. 1982. Kupferzeitliche Siedlungen in Nordostbulgarien. Munchen: C.H. Beck.

Todorova H. editor. 2002. Durankulak, Band II, Die prähistorischen Gräberfelder von Durankulak. Berlin-Sofia: Anubis Ltd.

Ursulescu N, Kogălniceanu R. 2007. Apparition des nécropoles dans le néolithique de Roumanie et de 1'Italie. Studia Antiqua et Archaeologica 12:11-42.

Wagner R. 1981. The Invention of Culture, Revised and expanded edition. Chicago and London: The University of Chicago Press.

Ward GK, Wilson SR. 1978. Procedures for comparing and combining radiocarbon age determinations: A critique. Archaeometry 20:19-31. 